\title{
A general spatio-temporal model of energy systems with a detailed account of transport and storage
}

\author{
Sheila Samsatlia,*, Nouri J. Samsatli ${ }^{\mathrm{a}}$ \\ ${ }^{a}$ Centre for Process Systems Engineering, Imperial College London \\ London SW7 2AZ, United Kingdom
}

\begin{abstract}
This paper presents a general spatio-temporal model of energy systems comprising technologies for generation/conversion, transport and storage and infrastructures for transport. The model determines the optimal network structure (e.g. location and size of technologies and their interconnections through transport infrastructures) and its operation (e.g. rate of utilisation of technologies and transport flows) considering simultaneously the short-term dynamics and a long-term planning horizon.
\end{abstract}

Here, we address one of the main challenges of solving a large scale MILP model: tractability. This issue is mainly caused by the need to include a wide range of time scales in the model: yearly (or decadal) intervals to include investment decisions; seasonal intervals to account for e.g. seasonal variations in demand and availability of resources; and hourly (or shorter) intervals to model the dynamics of storage technologies and to account for intermittency of renewable resources and demand. To exacerbate the problem, the spatial aspects also need to be fine enough to locate and size the technologies properly and to model the transport of resources, which depend on the location of demand and availability of resources. The model uses an efficient representation of time that exploits periodicity in system properties via a non-uniform hierarchical time discretisation. A decomposition method is also proposed wherein the large problem is broken down into 3 sub-problems that are then solved iteratively until the objective function is no longer improved. These methods significantly improve the computational efficiency without sacrificing temporal and spatial details.

The applicability of the model is illustrated using a case study in which the least-cost design and operation of a hydrogen network is determined such that the hourly transport demand of the different regions of an island is met by the intermittent and remotely-located wind energy.

Keywords: energy systems; energy storage and transport; Resource-Technology Network; spatio-temporal modelling; optimisation; hydrogen networks

\section{Introduction}

Spatio-temporal models account for dynamics and spatial dependence of system properties and are very important when modelling energy systems. This is because energy demands are not uniformly distributed and exhibit significant variations with time. Equally, primary energy resources are often localised and

\footnotetext{
${ }^{*}$ Corresponding author: Sheila Samsatli, email: s.samsatli@imperial.ac.uk, tel. +44(0)207594 3379
} 
intermittent in availability (e.g. wind power). In order to deploy assets strategically, it is important to understand the impact of different decisions relating to the size and location of different technologies for generation/conversion, storage and transport. Without a model that explicitly accounts for these spatial and temporal dependencies, it will not be possible to discriminate between alternative energy provision strategies (e.g. choosing between a centralised generation with a large transportation network versus distributed generation with lower transportation costs) or account for the dynamic nature of demands and resource availability. By considering the dynamics, the design and operation of energy storage technologies can be properly determined. Temporal aspects are also necessary for longer-term planning and strategic decision making.

One important area where spatio-temporal models can be applied is in modelling energy storage in the context of optimising a whole energy system. Indeed, energy storage is poorly represented in many pathway models used to plan for a low-carbon economy $[1,2]$ but is essential for ensuring a reliable supply of energy [3]. It is a key enabling element for the integration of variable resources such as wind and solar into the energy system $[4,5]$ and it can lower the cost of providing peaking generators and improve efficiency $[5,6]$.

The challenge of modelling energy storage in a whole energy system is mainly due to the complexity of 45 representing different technologies that operate across a wide range of timescales from seconds to years. Figure 1 shows the time scales over which the dynamics of storage technologies occur, which need to be captured by the model. While the dynamics of energy storage technologies occur over short time scales $(<$ hourly), the time horizon of energy system planning models is typically greater than one year. Therefore, a large number of time intervals is needed, which results in a very large model. To add to the challenge, 50 a spatially-distributed representation is also necessary to account for the distributed nature of primary energy sources and demands, transport of resources and to capture the trade-offs between a centralised and a distributed network. A whole energy system model will also include a large number of resources and technologies. This inevitably results in a very large and complex problem that is extremely challenging to solve. Indeed, tractability is the main issue associated with a spatio-temporal energy system model that includes storage. 


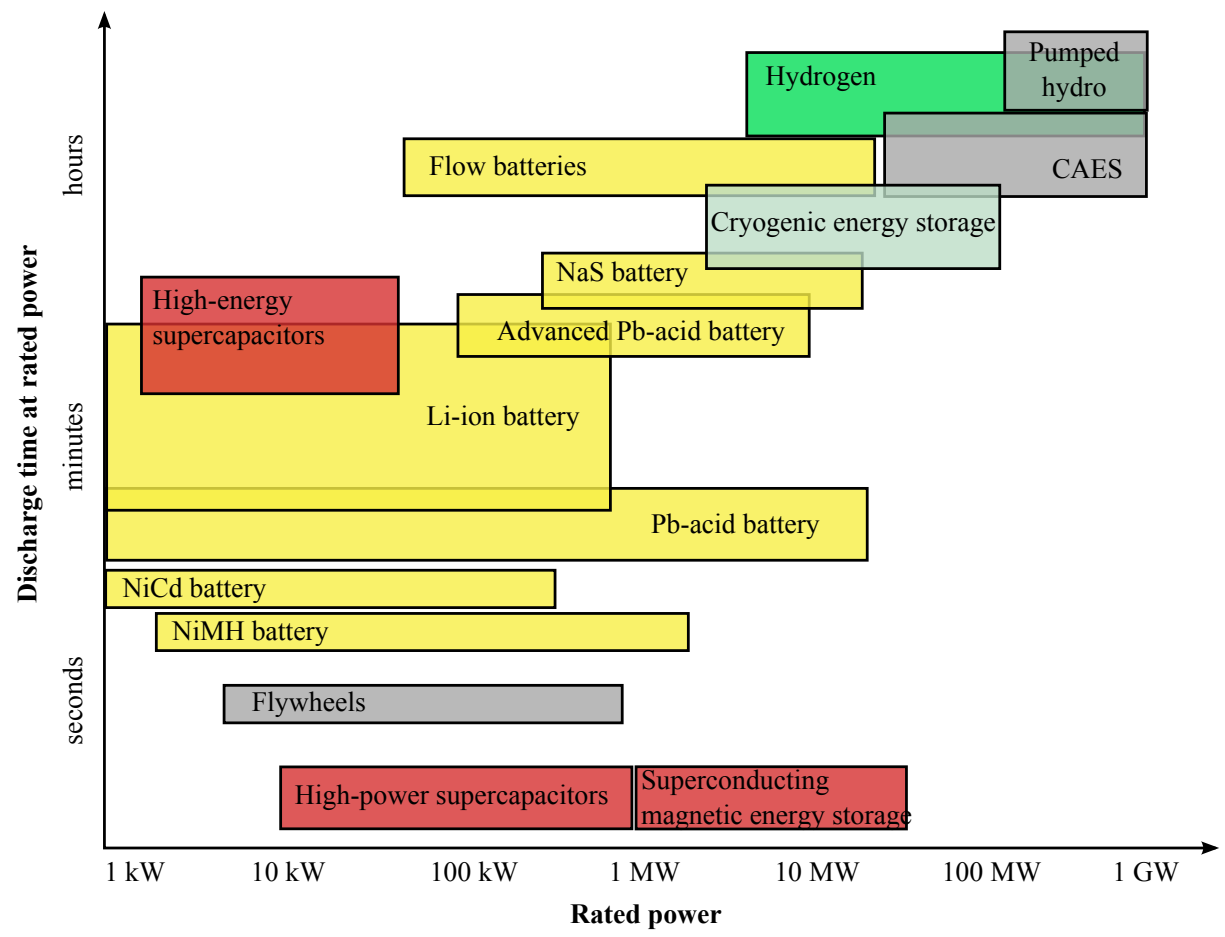

Figure 1: Different energy storage technologies and their discharge time at rated power (adapted from [7, 8]). Their dynamics, which occur over short time scales, need to be included in energy system models with long planning horizon resulting in a very large MILP model that is typically intractable.

In this paper, a general mixed-integer linear programming (MILP) model is presented that can represent energy storage in a whole energy system. The main contribution of the model is its capability in determining the optimal network structure (e.g. the location, number and size of generation/conversion and storage technologies and the transport infrastructures that interconnect them) and its operation (e.g. rate of operation of technologies and transport flows) considering simultaneously the short-term dynamics and a long-term planning horizon. This paper also presents two methods for addressing the tractability challenge that arises in solving a large scale MILP problem. The first method is a non-uniform hierarchical time discretisation, wherein the periodicity in system properties is exploited to represent time efficiently. The second approach is a decomposition method, where the main problem is decomposed into 3 sub-problems that are then solved iteratively until the objective function is no longer improved. Application of both methods to the problem results in a significant improvement in computational performance without sacrificing spatial and temporal details.

Different types of energy storage are currently available, which can be generally classified as chemical (e.g. gasoline, oil, hydrogen), mechanical (e.g. pumped hydro), electrochemical (e.g. batteries), electrical (e.g. capacitor, electromagnetic storage) and thermal (e.g. ice storage). In this paper, the applicability of the model is illustrated using an example problem of designing a hydrogen network comprising technologies for generation/conversion, storage and transport. Hydrogen is a promising energy carrier that is expected to be part of the future energy mix $[9,10,11]$. It can be viewed as an intermediate resource that can be converted to different energy vectors such as electricity, heat, transport fuel etc. Also, it is a flexible energy storage medium that can be used for both short- and long-term storage applications [12]. The main advantage of 
using hydrogen is it has no or very low local emissions.

Although applied only to hydrogen in the example, the model is general and applicable to the design of a network comprising any number of different energy carriers. Extending the model will simply involve including additional elements in the sets of resources, technologies etc. and all of the parameters (or data) associated with these elements. Similarly, changing the region of interest (e.g. to a country or a city) and time horizon is simply a matter of changing the data. This paper discusses in detail the mathematical formulation and considers an illustrative example of how the model can be used.

This paper is structured as follows: Section 2 surveys the relevant literature on spatio-temporal energy system modelling. Section 3 describes the five key elements of a spatio-temporal energy system model. The problem is defined in Section 4 and the Resource-Technology Network (RTN) used to represent the possible energy pathways in the problem is given in Section 5. Section 6 discusses the tractability issue arising from formulating the model in terms of contiguously hourly intervals and considering a long-term planning horizon - a simple spatio-temporal MILP model is presented to illustrate this issue. Section 7 describes the non-uniform hierarchical time discretisation approach. Section 8 gives the complete MILP formulation of the spatio-temporal model with detailed transport and storage and efficient representation of time. The decomposition method is presented in Section 9 to enhance further the computational efficiency of the model. The solution to the hydrogen network design problem is discussed in Section 10. Finally, some concluding remarks are made in Section 11.

\section{Literature review}

Energy security and climate change are the two main drivers of research in energy systems. Concurrent with these research activities is the work in modelling energy systems. There are many different models available in the literature. Jebaraj and Iniyan [13], Zeng et al. [14] and Bhattacharyya [15] surveyed many different energy system models, modelling approaches and their applications. Connoly et al. [16] analysed and compared different existing energy system models and concluded that there are no models that are suitable for all aspects of integrated renewable energy and therefore the tool of choice will depend on the specific scenario being considered.

Indeed, the choice of modelling approach depends on the questions one intends to answer. In this paper, we are particularly interested in optimisation models capable of representing systems that exhibit significant spatial (or geographical) and temporal dependencies. In general, spatial dependence is important for modelling transport and dynamic models are required for modelling storage.

Based on spatial resolution, energy models can be categorised as lumped or distributed (some authors described this as spatially-explicit). Lumped models have no spatial resolution at all and consider the whole region being modelled as a single entity; system properties are averaged or summed over space. Examples of such models are the widely-used MARKAL/TIMES $[17,18]$ policy planning models, which are based on an economic equilibrium framework. The strength of these models lies in their large set of technologies and their economic framework that can determine the equilibrium between supply and demand. However, because they are based on an LP formulation, they can only determine the level of penetration of each 
technology; they cannot determine the number and location of each technology, which requires an MILP formulation. They were not developed to determine the design and operation of the energy network, which

requires consideration of storage and transport, and therefore they have a very limited spatial and temporal resolution (see also Section 7). Other examples of lumped models include MESSAGE [19] and MODEST $[20,21]$. Distributed models explicitly account for spatial dependency and are usually employed in supply chain models (e.g. in $[22,23,24,25,26,27])$ and infrastructure planning models (e.g. in [28, 29]). The spatial element of the model is typically represented in one of two ways: by defining a set of nodes that represent key locations in the region of interest, e.g. candidate locations for technologies, and defining transport links between them (e.g. as was done in $[28,29]$ ); or by taking a more geographical view of the area and dividing the region into cells (e.g. as was done in $[22,23,24,25]$ ). The advantage of the former is that by limiting the number of sites at which technologies can be installed, the size of the model is more manageable. However, there is a possibility of overlooking a potential location and transport connections. Therefore, the latter approach is preferred because the optimiser can choose the most effective locations to place the technologies and design transport networks from among all of the possible transport connections.

Similarly, based on temporal resolution, models can be classified as steady-state, multiperiod or dynamic. Steady-state models do not account for variation with time and typically correspond to an average or peak scenario (e.g. the models in $[23,26]$ ). Multiperiod models essentially consider multiple steady-states (or periods) that represent different scenarios for the operation of the energy system (e.g. different levels of demand) but are coupled by the fact that the energy system design must be the same for all periods. For example, a model may consider a whole year of operation by dividing it into four periods: spring, summer, autumn and winter, each of which is considered as a separate steady-state scenario. The installed capacity of technologies is the same for each season but the operation may be different due to the different demands in each season. There are many multiperiod models where the periods represent intervals of a year or more (e.g. decades) and where the capacity of installed technologies in each period are linked by investment decisions and retirements of old technologies - there is a capacity balance constraint. Many future energy system models are multiperiod such as in [17, 18, 19, 20, 21, 24, 28], the peak/average version of the TURN model for urban energy systems design [30], and the BVCM [22] which reduces to a multiperiod model with staged investment when seasonality is not considered. Finally, dynamic models consider a much finer resolution for time, often using daily or shorter intervals, and are designed to look at short-term operational aspects of the energy system, such as how to account for storage (a dynamic inventory balance must be written) or how to account for the limited rate at which technologies can change load (hence ramp-up/ramp-down constraints are used and link the operation of the technologies from one time period to the next). Dynamic models may also include staged capacity investment but they are very rare due to the difficulty of including short-term dynamics over a long enough horizon to consider capacity planning - the models become very large and can be intractable.

Any model can then be classified according to its spatial and temporal resolution as described above, such as: lumped steady-state, distributed steady-state, through to distributed dynamic models. This last category is referred to here as spatio-temporal models.

As already mentioned, spatio-temporal models are required to determine the optimal location and size of storage technologies. Hoffman et al. [31] analysed the characteristics of a number of energy system models and concluded that most existing models and tools, both commercial and non-commercial, are focussed on 
determining system capacity, network design and location of generation technologies but none are suitable for sizing and locating energy storage. Energy storage modelling is an emerging area with most studies focussing on electricity storage $[32,33,34,35,36,37,38,29]$ and typically considering only short-term operation. All of them consider the spatial aspects by using a few pre-defined nodes. Bejan et al. [32] considered operation of generic large-scale storage for wind energy integration. They cover two weeks of operation at the hourly level. Borghetti et al. [33] and Khodayar et al. [34] developed a model to explore the operation of pumped hydro storage, considering 1 week at the hourly level. Parastegari et al. [35] developed a more general model but only considering operation over one day, also at the hourly level. A number of models have also been developed that optimise size and location of storage technologies but these are also limited by the time horizon considered: Carpinelli et al. [36] and Ghofrani et al. [37] are limited to a single day's operation at the hourly level; Nick et al. [38] also considered a single day with 24 hours but performed the analysis for winter and summer days separately. Pudjianto et al. [29] modelled the GB electricity system and optimised its operation with respect to storage and demand-side response for a time horizon of three days with 1-hour intervals.

Most of the models described in the previous paragraph are limited in their spatial resolution (a few predefined nodes are used) and the length of the time horizon (at most a week at the hourly level). They also represent pathways in an inflexible manner: the models are usually only applicable to the energy vector considered (electricity) and there are no interconversion pathways by which other energy vectors can be included. More flexible representations of energy conversion pathways have been developed in recent years. Geidl et al. [39, 40] developed the "energy hub" concept and the RTN approach was applied to whole system biomass value chains by Samsatli et al. [22] and to urban energy systems by Samsatli and Jennings [30].

Geidl $[39,40]$ introduced the concept of energy hubs in the context of optimising coupled energy infrastructures. The energy hub comprises a superstructure of converter elements, which convert one energy form to another with a given efficiency, and ideal storage modules (no storage loss). The storage modules are interfaced to the power flows in the hub via different charging and discharging efficiencies. The model of the energy hub is represented as a matrix of coupling coefficients that are derived from the power converter and storage efficiencies, dispatch variables (that define the flows between all elements in the hub) and binary variables that represent the existence of the power converters. As the converter efficiencies may depend on the power flows, the model results in a MINLP problem (it is not stated whether the dispatch variables appear multiplied by the binary variables but this could also be another source of non-linearity). A contiguous time representation is used to represent the dynamics of the system, with a cyclic constraint on the storage inventory. Some of the shortcomings of their approach are that the model is non-linear (therefore is harder to solve), the superstructure needs to be pre-defined, there is no explicit spatial representation (therefore the model cannot determine transport networks) and they only considered 12 1-hour intervals in their case study. In an extension of this work [41], robust optimisation of an energy hub was considered with 24 1-hour intervals but there was still no spatial representation.

The BVCM (Biomass Value Chain Model) [22] is a multi-vector spatio-temporal model for bioenergy. The purpose is to determine what crops to grow, when and where to grow them in order to convert them to various energy vectors. Therefore a high spatial resolution is required in order to account for effect of location on the yields of the biomass due to the different soil quality, precipitation levels etc. A high spatial resolution also allows the model to trade off decisions around transport and pre-processing of biomass feedstocks. As it is a 
pathway model to 2050, it needs to consider time at a coarse level (decades) but it can also capture seasonal variations in crop production: therefore it is a multiperiod model with staged invesment when seasonality is not considered and a dynamic model when seasonality and storage of resources are considered. As it is based on an RTN representation (see Figures 2 and 3 in [22] for illustrations), the energy pathways are not limited as they are in multi-echelon supply chain models (see also Section 2 of [22] for a discussion of RTN vs. multi-echelon representation). Given an overall target for energy production from biomass as a fraction of the total energy demand, the model can decide what form of energy (electricity, heat, transport fuel etc.) to produce for a given objective. Because the spatial resolution is so high, the temporal resolution must be limited: the solution time can be very high when seasonality and storage are considered.

Finally, the TURN model [30], developed by N. J. Samsatli, is a spatio-temporal multi-vector urban energy system model also based on the RTN representation. The tasks are similar to the converter elements in the energy hub but there is no need to specify a superstructure of tasks: the optimisation will determine the energy pathways depending on the scenario. It has an explicit representation of space and an efficient representation of time. As well as a fully dynamic formulation, there are multiperiod and steady-state versions. The latter has been used in a number of studies [42, 43, 44]. One limitation of the model is that, even with the efficient time representation, it can be difficult to solve problems involving high resolutions in space and time: the case study presented in [30] was very difficult to solve even with only 9 cells and 18 time intervals (6 hourly intervals for three seasons).

In summary, a wide variety of models have been presented in the literature. The model presented in this paper, STeMES, is aimed at solving a detailed planning and operation problem considering a high spatial and temporal resolution. It is therefore in the same category as the last few models described above: e.g. $[22,23,24,25,26,30,39,40]$. It was primarily developed in order to try to address some of the weaknesses of these models: that they are not able to consider transport and storage at an hourly level at the same time as considering long-term planning. The model presented here shares some of the features of the TURN model [30], such as using tasks to represent transport and storage and the efficient time representation. However, unlike TURN, which struggled to solve a problem with 9 cells and 18 time intervals, this new model is capable of modelling storage technologies at a temporal resolution sufficient to capture their dynamics. The spatial resolution is sufficiently fine to model transport of resources. Finally, it is based on the RTN representation, so the energy pathways are flexible and easily extensible to account for other energy vectors.

\section{Key elements of a spatio-temporal energy system model}

The model presented in this paper contains the following five key elements:

1. Space

The region of interest is divided into a number of cells, each of which which may be of any shape and size, represents a geographical location and is characterised by its centroid and area. The cells may have different dynamic demands for various resources and some resources may be available in certain cells in varying quantities. Cells can host technologies for conversion and storage of resources, they may be connected with other cells via transport infrastructures and may import or export resources. 


\section{Time}

The time element needs to capture long-term strategic decisions as well as short-term operational issues, such as intermittency and the dynamics of storage. The temporal resolution needs to be chosen in order to represent all of the system properties, such as demands, resource availability, prices etc., that depend on time.

\section{Resources}

Resources refer to any material or energy considered in the energy system model. A resource can be consumed or produced by a technology, transported from one cell to another cell, stored and/or imported from outside the region under study (e.g. from abroad) to certain locations. Resources include: raw materials or primary energy resources such as sunlight, wind or biomass; intermediates, which are produced by one technology and then consumed by a different technology, e.g. syngas, pyrolysis oil; end-use vectors such as electricity, heat and transport fuels (hydrogen and bio-methane are also widely regarded as final vectors); by-products, which are valuable materials that are generated in the process of producing the end-use vectors; and wastes, e.g. low-grade heat and emissions.

\section{Technologies}

Technologies represent devices for resource conversion, storage and transport. A technology may be available at different scales of capacity which could vary from household scale to industrial scale. They are characterised by a set of conversion factors that define how much of each resource is produced and consumed by the operation of a technology (this essentially defines the efficiency). Any resource can be an input to or an output from a technology, so that the optimisation can choose the most effective conversion pathway. The relationship between resources and technologies is described in more detail in Section 5.

(a) Generation/conversion

These technologies convert one set of resources into another set of resources. For example, a gas turbine takes natural gas and converts it into electricity, waste heat and $\mathrm{CO}_{2}$.

(b) Storage

These technologies represent devices or physical media that can accumulate (i.e. store and hold) a particular resource for some time and release it at a later time.

(c) Transport

These technologies move resources from one location to another using the transport infrastructures. Examples are trucks, tank trailers, barges, flow through piplines and so on.

The advantage of treating storage and transport as technologies is that it allows one to model resource requirements and losses. E.g., electricity required to operate storage devices and compressors/pumps to transport fluid along pipelines.

5. Transport infrastructures

Transport infrastructures connect different cells and are used by the transport technologies. Examples are roads and railways used to transport solid and liquid resources, electricity and natural gas grids, district heating networks, pipelines for e.g. hydrogen, $\mathrm{CO}_{2}$ and syngas. The reason for considering infrastructures explicitly is that some transport technologies may share the same infrastructure. For example, road tankers for petrol, LNG, hydrogen all use the same road infrastructure; if a new infrastructure is built, then all of the supported transport modes are enabled and there is no need to build separate infrastructures (which would overestimate the cost). 


\section{Problem description}

Given:

- A spatial description of the region under study represented by a number of cells. Each cell is characterised by:

- The coordinates of its centroid, which are used to calculate the distance between each cell

280

- Any restrictions on conversion and storage technologies that can be located in the cell

- Existence of a terminal for import/export of resources (e.g. ports)

- A set of resources, with each resource having the following properties:

- Demand as a function of space and time

- Availability as a function of space and time

- Unit cost and GHG emissions associated with its purchase, e.g. from the grid, which may depend on time and location

- A set of technologies that (when combined) can convert primary energy resources to final energy vectors, via any number of intermediates. Each technology is characterised by its:

- Minimum and maximum capacity

- Efficiency (in terms of conversion factors for input resources and output resources)

- Capital, operating and maintenance costs

- Maximum number that can be built each year for the whole region (also referred to as build rate)

- A set of storage technologies, each characterised by

- Efficiencies associated with charging, holding and discharging a resource (these combine to give the round-trip efficiency)

- Maximum capacity

- Minimum and maximum charge and discharge rate

- Unit capital impact (i.e. cost and GHG emissions)

- Unit operating impact associated with charging, holding and discharging a resource

- A set of transport technologies, each defined by its

- Minimum and maximum capacity

- Efficiency in transporting a resource (which can be dependent or independent of the distance travelled) 
- Unit cost and GHG emissions associated with transporting a resource (which can be dependent or independent of the distance travelled)

- A set of transport infrastructures, each characterised by its

- Maximum capacity

- Unit capital impact (i.e. cost and GHG emissions) for establishment

- If it is unidirectional, bidirectional, etc.

The development of the model is facilitated by an example, which illustrates many features that are typically encountered when modelling energy systems that have both temporal and spatial dependencies. The example considers a geographical region, shown in Figure 2(a), that represents an island using $1450 \mathrm{~km} \times 50 \mathrm{~km}$ squares. There is a plan to replace all fossil fuel powered cars with fuel cell cars running on hydrogen. There 330

- The number, location and size of production and storage technologies and transport infrastructures invested in every year

- The operation of each conversion technology (as a function of time)

- Utilisation rate of each technology (which gives the production/consumption rate of each resource)

- The operation of each storage technology (as a function of time)

- Rate at which a resource is added, held and withdrawn from each storage technology (which gives the inventory level of each resource in storage)

- The operation of the transport infrastructure (as a function of time)

- Rate of transport of each resource via each transport mode between all locations

- Meeting the energy demand for each resource in each cell at all times

- Maximum resource availability (local and imported)

- Maximum capacity of technologies (production, storage and transport)

In order to:

- Optimise some performance criterion (e.g. minimise total cost and/or GHG emissions)

are two wind farms, located in the north-east and south-west coasts of the island, which can be used to generate electricity that is then converted to hydrogen by electrolysis. The blue circles in the figure indicate 
the average daily demand for hydrogen for transport in each cell; it also shows the location (cell 9) of a salt cavern that may be used for hydrogen storage. Figure 2(b) shows the hourly transport demand along with the wind potential for a representative year (based on half-hourly historical data given in $[45,46]$ ). The transport demand has daily variations, being lower at weekends, as well as an overall seasonal variation, peaking in the summer. The wind potential is much more variable, sometimes higher and sometimes lower than the transport demand. In contrast to the transport fuel demand, the wind potential peaks during the winter months and is lowest during the summer. Both of these features suggest that storage could play a role in ensuring the demands are always satisfied.

340 The aim is to design a hydrogen infrastructure network comprising technologies for resource interconversion, storage and transport utilising the intermittent wind capacity to meet the temporally- and spatiallydistributed transport demand. The generated hydrogen can be transported to different cells and stored in either liquid or gaseous form. The potential storage technologies include compressed gaseous storage (e.g. pressurised tanks), liquid storage (e.g. cryogenic vessels), metal hydride and underground storage. The 345 problem involves determining the network components, their number, size and location, their interconnections (through transport infrastructures), and their hourly operation over an entire year so as to minimise the total system cost. 


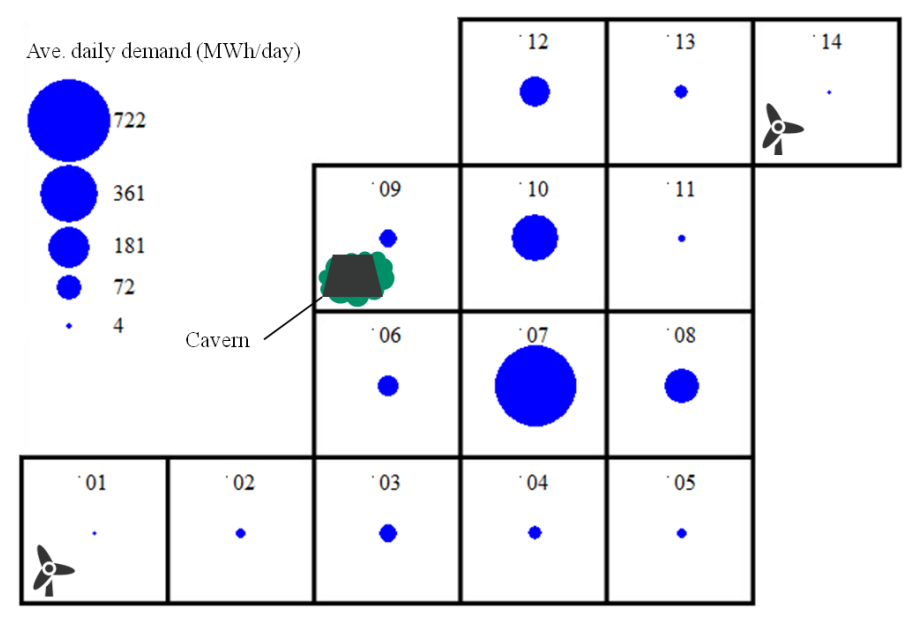

(a)

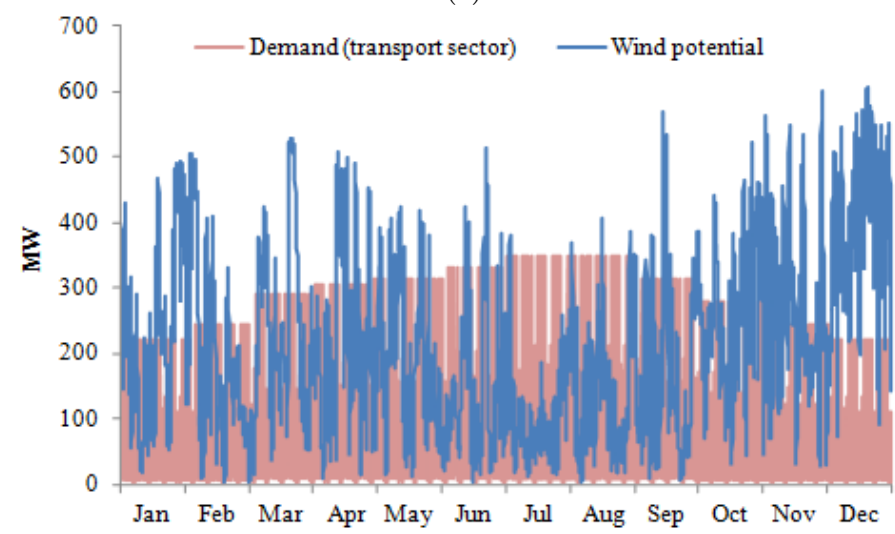

(b)

Figure 2: Example network design problem in which the spatially- and temporally- distributed transport fuel demand of an island is to be met by intermittent wind capacity: (a) geographical region under study discretised into 14 cells showing the distribution of transport demand over different locations in the island; (b) representative hourly data for transport energy demand and wind energy potential.

\section{Resource-Technology Network}

The possible energy pathways can be represented using the Resource-Technology Network, which is based on the State-Task Network (STN) approach that was first introduced by Kondili et al. [47]. The STN is a diagram used to represent batch chemical process recipes and comprises two types of node: states (usually drawn as circles) to represent any distinct material state (i.e. having a particular composition, temperature and pressure) and tasks (usually drawn as rectangles) to represent processes that convert a set of input states to a different set of output states. Pantelides [48] generalised this into Resource-Task Network for the description and solution of process scheduling problems: resources can represent any entities that are involved in the process (e.g. materials, equipment, utilities, equipment condition etc.). This evolved to the Resource-Technology Network (see Section 3 for the definition of "resource" and "technology") to represent different energy interconversion pathways of systems at national or regional scale. The concept of space was introduced by discretising the region of interest into a number of cells - the optimiser then determines the different RTNs in different cells. Whereas formulations based on the STN and the Resource-Task Network 
were written specifically to determine the schedule of batch operations in a multipurpose chemical plant, the purpose of the formulations based on the Resource-Technology Network is to determine the design and operation of energy networks. Examples of energy system models that employ RTN are the BVCM [22] for bioenergy systems and the TURN model [30] for urban energy systems.

The RTN representation of the hydrogen network design problem described in Section 4 is shown in Figure 3. Resources such as electricity, compressed gaseous hydrogen $\left(\mathrm{CGH}_{2}\right)$ and liquid hydrogen $\left(\mathrm{LH}_{2}\right)$ can be converted from one form to another via the following technologies: electrolyser, fuel cell, liquefier and regasifier. Each conversion is associated with an efficiency to account for losses. The resources can be stored in a variety of storage technologies and transported to or received from another location via different logistical modes: rail, truck and pipeline. The demands for any resource $\left(\mathrm{CGH}_{2}\right.$ in this particular problem), which is represented by a diamond in Figure 3, have to be satisfied. Finally, resources can be available locally (e.g. wind potential represented by the wind turbine in the diagram) or imported/exported.

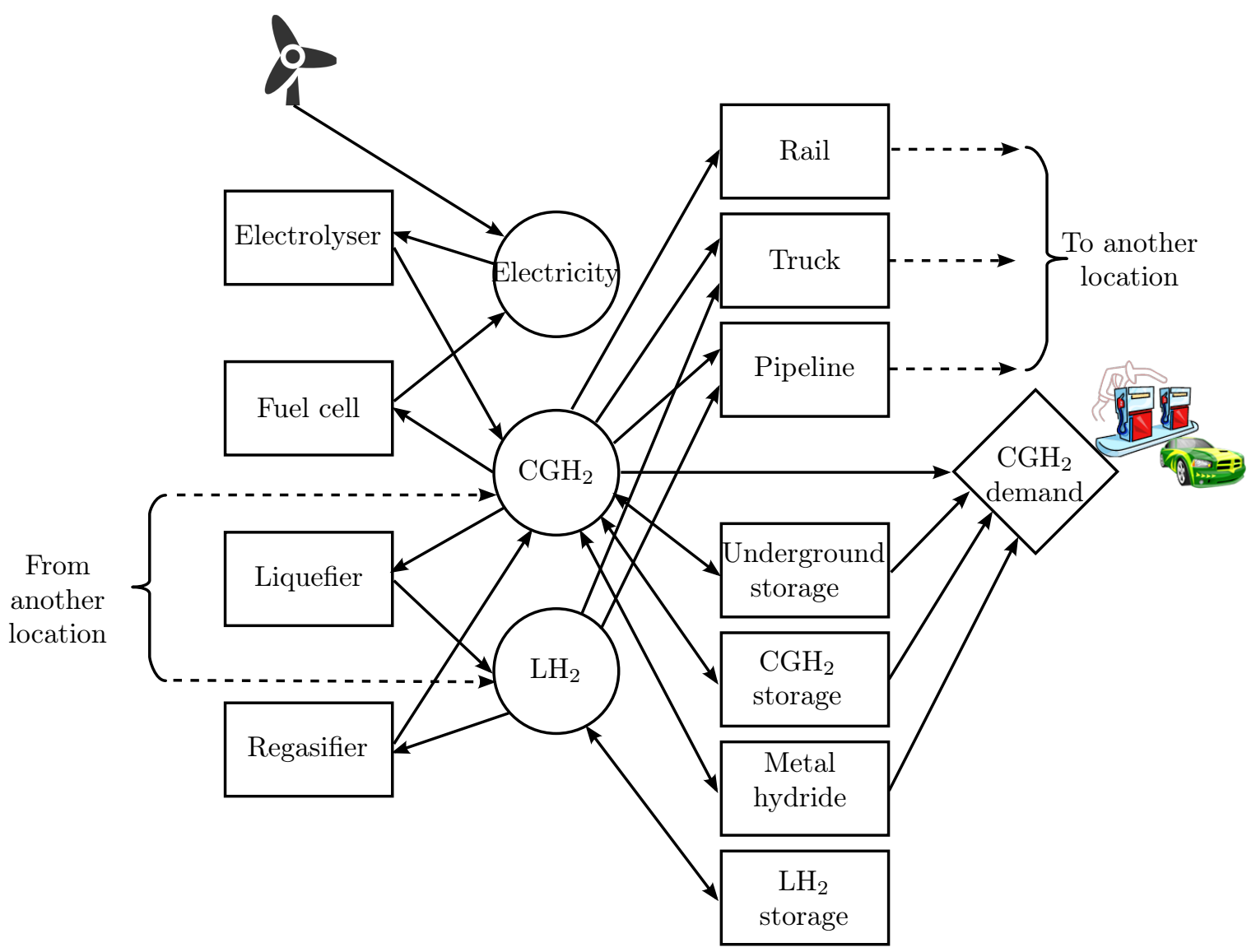

Figure 3: Resource-Technology Network for the hydrogen network design problem showing different energy interconversion pathways.

\section{The tractability challenge}

Before the detailed model is presented, the tractability issue arising from considering a long-term planning

horizon and formulating the model in terms of contiguous hourly intervals is first discussed. For this purpose, 
a simple spatio-temporal MILP model is presented without a detailed account of transport and storage, i.e. any resource can be transported up to a certain rate and stored up to a certain level without consideration of any specific mode or technology. Also, losses due to transport and storage are not considered. For simplicity, it is also assumed that the necessary transport infrastructures already exist. This is because modelling the investment decision for new transport infrastructures requires binary variables which add complication. The number of plants and storage facilities are modelled as integer variables.

Consider the following notation: $r$ is an element in the set of resources $\mathbb{R}$; $c$ is an element in the set of cells $\mathbb{C}$; $p$ is an element in the set of conversion plants $\mathbb{P}$; and $\tilde{h}$ is an element in the set of contiguous hourly intervals $\tilde{\mathbb{H}}$. The tilde in the notation indicates that the hourly intervals are contiguous.

Constraint 1 gives the resource balance, which states that for each resource $r$, in each cell $c$, at each hourly interval $\tilde{h}$, the local availability $U_{r c \tilde{h}}$, import from outside the region $M_{r c \tilde{h}}$, net production due to operation of conversion technologies $\sum_{p \in \mathbb{P}} \alpha_{r p} \mathscr{P}_{p c \tilde{h}}$ (where $\mathscr{P}_{p c \tilde{h}}$ is the rate of operation of technology $p$ and $\alpha_{r p}$ is the conversion factor defined as the net production of resource $r$ per unit operation of technology $p-$ $\alpha_{r p}$ is positive if $r$ is produced and negative if $r$ is consumed), and the net inflow from all other cells $\sum_{c^{\prime} \in \mathbb{C}}\left(Q_{r c^{\prime} c \tilde{h}}-Q_{r c c^{\prime} \tilde{h}}\right)$, must be equal to the demand $D_{r c \tilde{h}}$, export $X_{r c \tilde{h}}$ and the amount of resource that is transferred into storage $I_{r c \tilde{h}}-I_{r c, \tilde{h}-1} \cdot Q_{r c c^{\prime} \tilde{h}}$ is the rate of transport of resource $r$ from cell $c$ to cell $c^{\prime}$ at time $\tilde{h}$ and $I_{r c \tilde{h}}$ is the amount of resource $r$ in storage in cell $c$ at the end of time $\tilde{h}$.

$U_{r c \tilde{h}}+M_{r c \tilde{h}}+\sum_{p \in \mathbb{P}} \alpha_{r p} \mathscr{P}_{p c \tilde{h}}+\sum_{c^{\prime} \in \mathbb{C}}\left(Q_{r c^{\prime} c \tilde{h}}-Q_{r c c^{\prime} \tilde{h}}\right)=D_{r c \tilde{h}}+X_{r c \tilde{h}}+I_{r c \tilde{h}}-I_{r c, \tilde{h}-1} \quad \forall r \in \mathbb{R}, c \in \mathbb{C}, \tilde{h} \in \tilde{\mathbb{H}}$

Constraint 2 limits the production rate of each technology: the total rate of operation of all technologies of type $p$ in cell $c$ at time $\tilde{h}$ cannot exceed the maximum production rate of one technology $p_{p}^{\max }$, multiplied by the number of technologies of that type present in that cell, $N_{p c}^{P}$ (integer variable).

$$
\mathscr{P}_{p c \tilde{h}} \leq N_{p c}^{P} p_{p}^{\max } \quad \forall p \in \mathbb{P}, c \in \mathbb{C}, \tilde{h} \in \tilde{\mathbb{H}}
$$

If resource $r$ is locally-available in cell $c$, the rate of its utilisation, $U_{r c \tilde{h}}$, is limited by its maximum availability $u_{r c \tilde{h}}^{\max }:$

$$
U_{r c \tilde{h}} \leq u_{r c \tilde{h}}^{\max } \quad \forall r \in \mathbb{R}, c \in \mathbb{C}, \tilde{h} \in \tilde{\mathbb{H}}
$$

Constraint 4 ensures that the storage rate $I_{r c \tilde{h}}$, does not exceed the maximum storage capacity, which is the maximum capacity of one storage technology, $s_{r}^{\max }$, multiplied by the number of storage technologies, $N_{r c}^{S}$ (integer variable).

$$
I_{r c \tilde{h}} \leq N_{r c}^{S} s_{r}^{\max } \quad \forall r \in \mathbb{R}, c \in \mathbb{C}, \tilde{h} \in \tilde{\mathbb{H}}
$$


Similarly, constraints 5 and 6 limit the rate of import and export, respectively, of resource $r$ in cell $c$ at time $\tilde{h}$ :

$$
\begin{aligned}
& M_{r c \tilde{h}} \leq m_{r c \tilde{h}}^{\max } \quad \forall r \in \mathbb{R}, c \in \mathbb{C}, \tilde{h} \in \tilde{\mathbb{H}} \\
& X_{r c \tilde{h}} \leq x_{r c \tilde{h}}^{\max } \quad \forall r \in \mathbb{R}, c \in \mathbb{C}, \tilde{h} \in \tilde{\mathbb{H}}
\end{aligned}
$$

Finally, equation 7 gives the objective function, which is a measure of the overall performance of the system, e.g. economic and environmental impacts, expressed in terms of the decision variables in the model:

$$
Z=f\left(M_{r c \tilde{h}}, U_{r c \tilde{h}}, \mathscr{P}_{p c \tilde{h}}, Q_{r c c^{\prime} \tilde{h}}, X_{r c \tilde{h}}, I_{r c \tilde{h}}, N_{p c}^{P}, N_{r c}^{S}\right)
$$

405

410

The tractability of the model depends on the total number of variables and constraints - many of these depend on position and time (indices $c$ and $\tilde{h}$ in the model). There is a trade-off between the number of cells and time intervals (which determine the fidelity of the model) and the ease with which the model can be solved. Using a very fine temporal and/or spatial discretisation results in a very large model that is difficult to solve in an acceptable amount of time. On the other hand, aggregation to a coarser resolution often results in loss of details, e.g. smoothing-out of the variability in the data, which can affect decisions such as scale and location of production and storage technologies as well as the structure and operation of the transport network.

The model described by constraints 1 to 7 was applied to the hydrogen network design problem described in Section 4. In the problem, $|\mathbb{R}|=3,|\mathbb{P}|=4,|\mathbb{C}|=14$ and there are 84 integer variables (for simplicity, only storage of $\mathrm{CGH}_{2}$ and $\mathrm{LH}_{2}$ is considered). The planning horizon, $|\tilde{\mathbb{H}}|$, is gradually increased, from one day (with 24 hourly intervals) to one year (with 8,760 hourly intervals), to determine at what point the model becomes intractable. Table 1 gives the number of variables and constraints in the model for different lengths of planning horizon and the time it takes to obtain a full integer solution. The model was implemented in AIMMS 3.12 and each optimisation was solved to a relative tolerance of $0.1 \%$ using CPLEX 12.5 on a PC with an Intel Xeon CPU at $3.19 \mathrm{GHz}$ and $48 \mathrm{~GB}$ of RAM.

Table 1: Benchmarking of the model described by constraints 1 to 7 .

\begin{tabular}{rrrr}
\hline No. of contiguous hourly intervals, $|\tilde{\mathbb{H}}|$ & No. of variables & No. of constraints & CPU time (s) \\
\hline 24 & 14,203 & 6,391 & 18 \\
168 & 98,875 & 44,695 & 679 \\
720 & 423,451 & 191,527 & 47,955 \\
2,160 & $1,270,171$ & 574,567 & Intractable \\
8,760 & $5,150,971$ & $2,330,167$ & Intractable \\
\hline
\end{tabular}

Table 1 shows that the model size increases (almost) linearly with the length of the planning horizon but the solution time increases (almost) exponentially. A solution was not obtained for the run with 2,160 hourly intervals after 5 days: the model seems to be intractable already. Reducing the number of cells can help improve tractability, e.g. with only 6 cells a solution was obtained for a full time horizon of 8,760 hours after 
approximately 3 days. However, 6 cells are probably not sufficient to represent all of the spatial features of the problem, e.g. the resulting transport network may be smaller than what is actually needed.

Of course, this is just a simple model, so adding more complexity, such as detailed storage and transport, more resources and technologies to represent more energy pathways, is only going to make the tractability issue worse. There are two obvious ways to reduce the size of the problem: use fewer, larger cells or use fewer, longer time intervals. Both of these result in loss of accuracy. However, instead of using longer time intervals, one can take advantage of the periodicity in the data with respect to time. The problem can also be decomposed into several stages and solved iteratively until the best solution is found. The non-uniform hierarchical time discretisation and a decomposition method are discussed in the subsequent sections. These methods can reduce the solution time and allow more details to be included in the model, e.g. different types of transport mode and storage technology, losses due to transport and storage, a more detailed account of storage operation and investment in transport infrastructures.

\section{Non-uniform hierarchical time discretisation}

The non-uniform hierarchical time discretisation involves decomposition of the time domain into different sub-domains of varying granularity. The time domain is divided into hourly intervals $h \in \mathbb{H}$, daily intervals $d \in \mathbb{D}$, seasonal intervals $t \in \mathbb{T}$, and yearly intervals $y \in \mathbb{Y}$, as illustrated in Figure 4. When applying this approach to model storage, it is important to link inventories within and between time levels, therefore additional variables are needed for the initial inventories and additional constraints are required to link the inventories - these are all given by equations 16 to 25 .

The hourly intervals can be non-uniform, each having a duration of $n_{h}^{h d}$ hours, such that $\sum_{h} n_{h}^{h d}=24$. For example, daily demand profiles can be represented more efficiently by using more short-duration intervals during peak times to capture the dynamics and fewer longer-duration intervals during off-peak times when the demand is relatively static.

The daily intervals can be represented more efficiently by exploiting the periodicity in the demand data. For example, weekdays are likely to have similar demand profiles, which are likely to be different to those for weekend days. Instead of using 7 daily intervals per week, a particular day type can be repeated a certain number of times. The demands can be modelled as a sequence of repeated profiles: a week's worth of data can be represented by 5 repetitions of a weekday profile, followed by 2 repetitions of a weekend profile. Therefore, in the model each daily profile $d$ is repeated to give a total of $n_{d}^{d w}$ profiles, such that $\sum_{d} n_{d}^{d w}=7$.

Similarly, each season can be represented by a certain number of identical weeks. In the model each seasonal interval $t$ comprises $n_{t}^{w t}$ identical weeks, such that $\sum_{t} n_{t}^{w t}=52$.

Finally, the yearly interval is the level at the which investment decisions are typically included. Each yearly interval $y$ is composed of $n_{y}^{y y}$ identical years.

Overall, this discretisation results in a significantly fewer total time intervals: $|\mathbb{H}| \times|\mathbb{D}| \times|\mathbb{T}| \times|\mathbb{Y}|$. For example, for a whole year with 4 different season types, 2 days types and 24 hours in a day, the total number of time intervals is only 192, compared to 8,760 intervals if time is modelled using contiguous hourly intervals. 
By applying the non-uniform hierarchical time discretisation, the ability to capture accurately dynamics that are not periodic may be lost and the degree to which the hourly intervals can be simplified depends on the data (if they are not strongly periodic, then there may be no opportunity to reduce the hourly intervals in each day to fewer than 24). For example, in the case study presented here, the intermittent wind availability showed no consistent profile from day to day, so it was necessary to include all 24 hourly intervals. If wind were not considered, it may have been possible to reduce the number of time intervals if all of the demands and other temporally-dependent input data were roughly constant over the same period (e.g. overnight).

This approach may appear similar to the "time slicing" mentioned in the TIMES Model Documentation [17] and the mathematical formulation for the ETSAP-TIAM [49]: an index exists for time intervals of less than a year but is not enabled by default and must be configured by the user. One instance where this feature was used is the Temporal MARKAL model [50], where five intervals per day and four seasons were considered. The key difference between their approach and the one presented here is that each set of intervals can repeat and can be linked with the others. This allows the inventory to be tracked over the whole time horizon in an efficient way - indeed, the approach was developed specifically for dynamic models such as the one presented in this paper. Temporal MARKAL is a multiperiod model and therefore cannot model the inventory of stored resources. Storage is only considered by the user specifying one time interval in the day where resources can be stored and this allows some of the demands in the other intervals to be shifted to the one specified for storage.

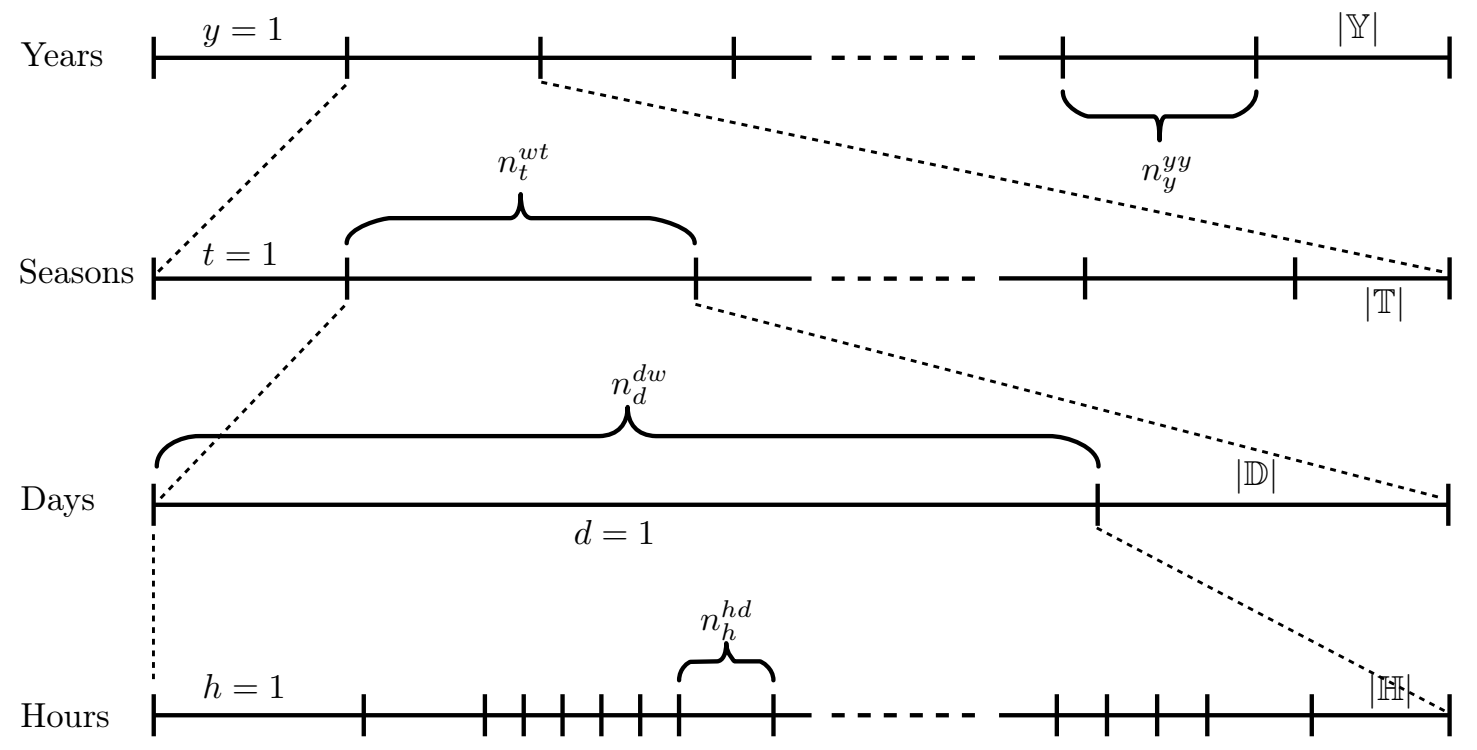

Figure 4: Non-uniform hierarchical time discretisation

\section{MILP formulation with efficient time representation}

Again, the starting point for the model is the resource balance given by constraint 8. Most symbols correspond exactly to those in equation 1 but with a non-uniform hierarchical time discretisation $(\tilde{h} \rightarrow h d t y)$. The third and fourth terms in equation 1 are replaced by the equivalent symbols $P_{r c h d t y}$ and $Q_{r c h d t y}$, defined similarly later. Since there is a more detailed model of resource storage, the inventory terms in equation 1 are replaced by $S_{\text {rchdty }}$, which is the net rate of utilisation of stored resource. 
$U_{\text {rchdty }}+M_{\text {rchdty }}+P_{\text {rchdty }}+Q_{\text {rchdty }}+S_{\text {rchdty }} \geq D_{\text {rchdty }}+X_{\text {rchdty }} \quad \forall r \in \mathbb{R}, c \in \mathbb{C}, h \in \mathbb{H}, d \in \mathbb{D}, t \in \mathbb{T}, y \in \mathbb{Y}$

\subsection{Resource availability}

Resource availability is restricted using the equivalent of constraint 3 for the non-uniform hierarchical time discretisation:

$$
U_{r c h d t y} \leq u_{r c h d t y}^{\max } \quad \forall r \in \mathbb{R}, c \in \mathbb{C}, h \in \mathbb{H}, d \in \mathbb{D}, t \in \mathbb{T}, y \in \mathbb{Y}
$$

\subsection{Resource conversion}

Resource conversion is also modelled equivalently to the simple model. The net production of resource (third term in constraint 1) and the upper bound on the production rate (constraint 2) are:

$$
P_{\text {rchdty }}=\sum_{p \in \mathbb{P}} \alpha_{r p} \mathscr{P}_{\text {pchdty }} \quad \forall r \in \mathbb{R}, c \in \mathbb{C}, h \in \mathbb{H}, d \in \mathbb{D}, t \in \mathbb{T}, y \in \mathbb{Y}
$$

and

$$
\mathscr{P}_{\text {pchdty }} \leq N_{p c}^{P} p_{p}^{\max } \quad \forall p \in \mathbb{P}, c \in \mathbb{C}, h \in \mathbb{H}, d \in \mathbb{D}, t \in \mathbb{T}, y \in \mathbb{Y}
$$

In addition, the minimum production capacity of plant $p$ in cell $c$ is given by constraint 12 , where $p_{p}^{\min }$ is the minimum capacity of one technology $p$.

$$
\mathscr{P}_{p c h d t y} \geq N_{p c}^{P} p_{p}^{\min } \quad \forall p \in \mathbb{P}, c \in \mathbb{C}, h \in \mathbb{H}, d \in \mathbb{D}, t \in \mathbb{T}, y \in \mathbb{Y}
$$

The limit on the total number of technologies of type $p$ that can be built over the entire region is given by constraint 13 , where $B R_{p}$ is the maximum number of plants $p$ (sometimes referred to as the build rate).

$$
\sum_{c \in \mathbb{C}} N_{p c}^{P} \leq B R_{p} \quad \forall p \in \mathbb{P}
$$




\subsection{Resource storage}

The process of storing a resource involves three stages: charging, maintaining and discharging. These are modelled using 3 storage tasks: "put", "hold" and "get". This approach allows different efficiencies, resource requirements, wastes, emissions and costs to be applied to each stage of storage. Figure 5 shows an example set of storage tasks for storing resource $r_{1}$. The "put" task transfers $r_{1}$ from the cell to the store, requiring some $r_{2}$ and producing some waste $r_{3}$ (e.g. $\mathrm{CO}_{2}$ or waste heat). The "hold" task maintains $r_{1}$ in the storage at less than $100 \%$ efficiency, the losses being converted to $r_{3}$; this stage also requires some $r_{2}$. Finally, the "get" task retrieves $r_{1}$ from the storage and delivers it to the cell, requiring some $r_{4}$.

For example, compressed gaseous hydrogen storage and underground storage require a "put" task involving a compression process. For liquid hydrogen storage, a "hold" task is needed to maintain the liquid hydrogen at a very low temperature to prevent boil-off; losses due to boil-off can be considered by specifying the efficiency of the "hold" task. For metal hydride storage, the adsorption process is the "put" task while the desorption process (i.e. the use of steam to desorb the hydrogen molecules from the metal) is the "get" task. For metal hydride storage, the "get" task is the most difficult and expensive stage [51].

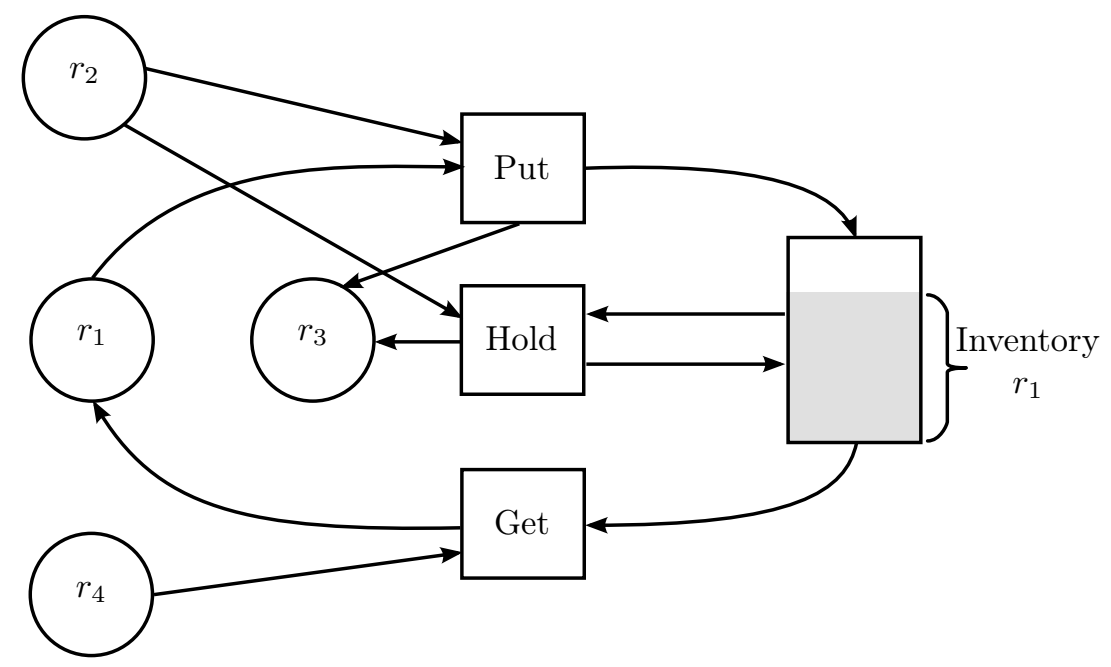

Figure 5: An example set of storage tasks for storing resource $r_{1}$.

510 To model the storage tasks, let $\mathscr{S}_{\text {schdty }}^{\text {put }}, \mathscr{S}_{\text {schdty }}^{\text {hold }}$ and $\mathscr{S}_{\text {schdty }}^{\text {get }}$ be positive variables representing the rate at which a resource is added, held and withdrawn, respectively, from a storage technology $s$ located in cell $c$ at time $h, d, t$ and $y$. Another set needs to be defined in order to describe the direction of the flow of resources, e.g. from the cell to the storage and vice versa: let $f$ represent an element in the set of flow directions $\mathbb{F} \equiv\{$ src, dest $\}$ (abbreviations for "source" and "destination", repectively). In the resource balance given by constraint 8, the term $S_{r c h d t y}$ is a free variable representing the net rate of utilisation of stored resource $r$, which is given by equation 14 in terms of the rate of each storage task.

$S_{r c h d t y}=\sum_{s \in \mathbb{S}}\left(\sigma_{s r, \text { src }}^{\text {put }} \mathscr{S}_{\text {schdty }}^{\text {put }}+\sigma_{s r, \mathrm{dst}}^{\text {hold }} \mathscr{S}_{\text {schdty }}^{\text {hold }}+\sigma_{\text {sr, dst }}^{\text {get }} \mathscr{S}_{\text {schdty }}^{\text {get }}\right) \quad \forall r \in \mathbb{R}, c \in \mathbb{C}, h \in \mathbb{H}, d \in \mathbb{D}, t \in \mathbb{T}, y \in \mathbb{Y}$ 
Here, $\sigma_{s r f}^{\text {put }}$ is the conversion factor when storing inventory (the source is the cell and the destination is the storage device), $\sigma_{s r f}^{\text {hold }}$ is the conversion factor when holding inventory and $\sigma_{s r f}^{\text {get }}$ is the conversion factor when withdrawing inventory (in these last two cases, the source is the storage device and the destination is the cell).

The inventory level in storage $s$ located in cell $c$ at the end of interval $h$ in day $d$, season $t$ and yearly interval $y$, represented by the positive variable $I_{\text {schdty }}$, is given by the inventory balance in equation 15 . Note that the subscripts for the flow directions are now the opposite of those that appear in equation 14.

$I_{s c h d t y}=n_{h}^{h d} \sum_{r \in \mathbb{R}}\left(\sigma_{s r, \mathrm{dst}}^{\mathrm{put}} \mathscr{S}_{\text {schdty }}^{\mathrm{put}}+\sigma_{s r, \mathrm{src}}^{\text {hold }} \mathscr{S}_{\text {schdty }}^{\text {hold }}+\sigma_{s r, \mathrm{src}}^{\text {get }} \mathscr{S}_{\text {schdty }}^{\mathrm{get}}\right) \quad \forall s \in \mathbb{S}, c \in \mathbb{C}, h \in \mathbb{H}, d \in \mathbb{D}, t \in \mathbb{T}, y \in \mathbb{Y}$

The initial amount held in the storage is given by equation 16 . Here, $I_{\text {scdty }}^{0 \text {,sim }}$ is a positive variable (i.e. one of 525 the start of a "simulated cycle" for day type $d$ in season $t$, yearly interval $y$. The simulated cycle is one day of operation for the particular day in day type $d$, week in season $t$ and year in yearly interval $y$ in which the hourly inventory profile is the same as the average hourly invetory profile over all days, weeks and years in the day type $d$, season $t$ and yearly interval $y$ (rather than the first day, which is represented by the "act" variables). This allows operating costs and resource requirements, both of which depend on the inventory levels to be calculated correctly. See also the detailed discussion in the appendix.

$$
\mathscr{S}_{s c, 1, d t y}^{\text {hold }}=I_{s c d t y}^{0, \text { sim }} / n_{1}^{h d} \quad \forall s \in \mathbb{S}, c \in \mathbb{C}, d \in \mathbb{D}, t \in \mathbb{T}, y \in \mathbb{Y}
$$

For $h>1$, the rate at which inventory is held can be expressed in terms of the inventory level divided by the duration of the hourly interval $h$ :

$$
\mathscr{S}_{\text {schdty }}^{\text {hold }}=I_{s c, h-1, d t y} / n_{h}^{h d} \quad \forall s \in \mathbb{S}, c \in \mathbb{C}, h>1 \in \mathbb{H}, d \in \mathbb{D}, t \in \mathbb{T}, y \in \mathbb{Y}
$$

The surplus over a day, $\delta_{s c d t y}$, is equal to the inventory at the end of the last hourly interval of the day, $|\mathbb{H}|$, minus the inventory at the beginning of the first interval of the day:

$$
\delta_{s c d t y}=I_{s c,|\mathbb{H}|, d t y}-I_{\text {scdty }}^{0, \text { sim }} \quad \forall s \in \mathbb{S}, c \in \mathbb{C}, d \in \mathbb{D}, t \in \mathbb{T}, y \in \mathbb{Y}
$$

The surplus over one week, $\delta_{\text {scty }}$, is the daily surplus multiplied by the number of times day $d$ occurs in a week, and then summed over all day types $d$ :

$$
\delta_{s c t y}=\sum_{d \in \mathbb{D}} \delta_{s c d t y} n_{d}^{d w} \quad \forall s \in \mathbb{S}, c \in \mathbb{C}, t \in \mathbb{T}, y \in \mathbb{Y}
$$


The surplus over one year, $\delta_{s c y}$, is the weekly surplus multiplied by the number of repeated weeks in season $t$, and then summed over all season types $t$ :

$$
\delta_{s c y}=\sum_{t \in \mathbb{T}} \delta_{s c t y} n_{t}^{w t} \quad \forall s \in \mathbb{S}, c \in \mathbb{C}, y \in \mathbb{Y}
$$

540

Note that the surpluses, $\delta_{s c d t y}, \delta_{s c t y}$ and $\delta_{s c y}$ are free variables (i.e. can be positive or negative).

The (optional) cyclic constraint given by equation 21 ensures that there is no accumulation of resource in storage over a year:

$$
\delta_{s c y}=0 \quad \forall s \in \mathbb{S}, c \in \mathbb{C}, y \in \mathbb{Y}
$$

Equation 22 links the inventories between day types. Here, $I_{\text {scdty }}^{0, \text { act }}$ is a positive variable (i.e. another degree of freedom) representing the (actual) inventory of storage $s$ in cell $i$ at the start of the first instance of day $d$ in the first week of season $t$, in the first year of yearly interval $y$.

$$
I_{s c d t y}^{0, \text { act }}=I_{s c, d-1, t y}^{0, \text { act }}+n_{d-1}^{d w} \delta_{s c, d-1, t y} \quad \forall s \in \mathbb{S}, c \in \mathbb{C}, d>1 \in \mathbb{D}, t \in \mathbb{T}, y \in \mathbb{Y}
$$

Equations 23 and 24 link the inventories between seasons and years, respectively:

$$
\begin{gathered}
I_{s c, 1, t y}^{0, \text { act }}=I_{s c, 1, t-1, y}^{0, \text { act }}+n_{t-1}^{w t} \delta_{s c, t-1, y} \quad \forall s \in \mathbb{S}, c \in \mathbb{C}, t>1 \in \mathbb{T}, y \in \mathbb{Y} \\
I_{s c, 1,1, y}^{0, \text { act }}=I_{s c, 1,1, y-1}^{0, \text { act }}+n_{y-1}^{y y} \delta_{s c, y-1} \quad \forall s \in \mathbb{S}, c \in \mathbb{C}, y>1 \in \mathbb{Y}
\end{gathered}
$$

Equation 25 (which is derived in the appendix) relates the starting inventory, $I_{\text {scdty }}^{0, \text { act }}$, and the simulated cycle, $I_{s c d t y}^{0, \operatorname{sim}}$ :

$I_{\text {scdty }}^{0, \text { sim }}=I_{\text {scdty }}^{0, \text { act }}+\left[\left(n_{d}^{d w}-1\right) \delta_{\text {scdty }}+\left(n_{t}^{w t}-1\right) \delta_{\text {scty }}+\left(n_{y}^{y y}-1\right) \delta_{s c y}\right] / 2 \quad \forall s \in \mathbb{S}, c \in \mathbb{C}, d \in \mathbb{D}, t \in \mathbb{T}, y \in \mathbb{Y}$

It is now necessary to ensure that the inventory in cell $c$ at any time does not exceed the maximum storage capacity in that cell, which is the maximum capacity of one storage technology of type $s, s_{s}^{\text {hold,max }}$, multiplied by the number of storage technologies of that type present in that cell, $N_{s c}^{S}$. Instead of writing the constraints for every (contiguous) hour, one can exploit the periodicity in system properties: the inventory increases or reduces depending on the number of repeated time intervals. Therefore, one only need to write the constraints for the first and last instance of each repeated day type, the first and last week of each season and the first and last season of each year. These are all given by constraints 26 to 33, in which the domain 
$\forall s \in \mathbb{S}, c \in \mathbb{C}, h \in \mathbb{H}, d \in \mathbb{D}, t \in \mathbb{T}, y \in \mathbb{Y}$ is omitted for brevity. The binary parameter $a_{s c}$ has a value of 1 when a storage technology $s$ can be installed in cell $c$ and 0 otherwise (e.g. this can be used to indicate the location of caverns for underground storage).

$$
\begin{gathered}
I_{s c h d t y}+\left[\left(n_{d}^{d w}-1\right) \delta_{s c d t y}+\left(n_{t}^{w t}-1\right) \delta_{s c t y}+\left(n_{y}^{y y}-1\right) \delta_{s c y}\right] / 2 \leq s_{s}^{\text {hold,max }} N_{s c}^{S} a_{s c} \\
I_{s c h d t y}+\left[-\left(n_{d}^{d w}-1\right) \delta_{s c d t y}+\left(n_{t}^{w t}-1\right) \delta_{s c t y}+\left(n_{y}^{y y}-1\right) \delta_{s c y}\right] / 2 \leq s_{s}^{\text {hold,max }} N_{s c}^{S} a_{s c} \\
I_{s c h d t y}+\left[\left(n_{d}^{d w}-1\right) \delta_{s c d t y}-\left(n_{t}^{w t}-1\right) \delta_{s c t y}+\left(n_{y}^{y y}-1\right) \delta_{s c y}\right] / 2 \leq s_{s}^{\text {hold,max }} N_{s c}^{S} a_{s c} \\
I_{s c h d t y}+\left[\left(n_{d}^{d w}-1\right) \delta_{s c d t y}+\left(n_{t}^{w t}-1\right) \delta_{s c t y}-\left(n_{y}^{y y}-1\right) \delta_{s c y}\right] / 2 \leq s_{s}^{\text {hold,max }} N_{s c}^{S} a_{s c} \\
I_{s c h d t y}+\left[\left(n_{d}^{d w}-1\right) \delta_{s c d t y}-\left(n_{t}^{w t}-1\right) \delta_{s c t y}-\left(n_{y}^{y y}-1\right) \delta_{s c y}\right] / 2 \leq s_{s}^{\text {hold,max }} N_{s c}^{S} a_{s c} \\
I_{s c h d t y}+\left[-\left(n_{d}^{d w}-1\right) \delta_{s c d t y}+\left(n_{t}^{w t}-1\right) \delta_{s c t y}-\left(n_{y}^{y y}-1\right) \delta_{s c y}\right] / 2 \leq s_{s}^{\text {hold,max }} N_{s c}^{S} a_{s c} \\
I_{s c h d t y}+\left[-\left(n_{d}^{d w}-1\right) \delta_{s c d t y}-\left(n_{t}^{w t}-1\right) \delta_{s c t y}+\left(n_{y}^{y y}-1\right) \delta_{s c y}\right] / 2 \leq s_{s}^{\text {hold,max }} N_{s c}^{S} a_{s c} \\
I_{s c h d t y}+\left[-\left(n_{d}^{d w}-1\right) \delta_{s c d t y}-\left(n_{t}^{w t}-1\right) \delta_{s c t y}-\left(n_{y}^{y y}-1\right) \delta_{s c y}\right] / 2 \leq s_{s}^{\text {hold,max }} N_{s c}^{S} a_{s c}
\end{gathered}
$$

The above contraints are also written with the left-hand side greater than or equal to zero to ensure that the inventory is always non-negative (not shown here for brevity).

Finally, the rate of each storage task (i.e. charging, maintaining and discharging) is limited by the maximum rate at which each task can be performed (constraints 34 to 36 ).

$$
\begin{array}{rlr}
\mathscr{S}_{\text {schdty }}^{\text {put }} \leq N_{s c}^{S} s_{s}^{\text {put,max }} a_{s c} & \forall s \in \mathbb{S}, c \in \mathbb{C}, h \in \mathbb{H}, d \in \mathbb{D}, t \in \mathbb{T}, y \in \mathbb{Y} \\
I_{\text {schdty }} \leq N_{s c}^{S} s_{s}^{\text {hold,max }} a_{s c} & \forall s \in \mathbb{S}, c \in \mathbb{C}, h \in \mathbb{H}, d \in \mathbb{D}, t \in \mathbb{T}, y \in \mathbb{Y} \\
\mathscr{S}_{\text {schdty }}^{\text {get }} \leq N_{s c}^{S} s_{s}^{\text {get,max }} a_{s c} & \forall s \in \mathbb{S}, c \in \mathbb{C}, h \in \mathbb{H}, d \in \mathbb{D}, t \in \mathbb{T}, y \in \mathbb{Y}
\end{array}
$$

\subsection{Resource transport}

Transport is also modelled as a task to allow for losses to be considered as well as to account explicitly for resource requirements when transporting a resource (e.g. fuel for road transport). If desired, GHG emissions or waste heat can also be tracked using this approach. Figure 6 shows an example transport task in which resource $r_{2}$ is transported from cell $c$ to cell $c^{\prime}$ requiring resource $r_{1}$ from cell $c$ and resulting in waste (e.g. GHG emissions) being generated in both cells.

In the resource balance, given by constraint $8, Q_{\text {rchdty }}$ is the net rate of transport of resource $r$ into cell $c$

$$
\begin{array}{r}
Q_{r c h d t y}=\sum_{c^{\prime} \mid \nu_{c^{\prime} c}=1} \sum_{l \in \mathbb{L}}\left[\left(\bar{\tau}_{l r, \mathrm{dst}}+\hat{\tau}_{l r, \mathrm{dst}} d_{c^{\prime} c}\right) \mathscr{Q}_{l c^{\prime} c h d t y}\right]+\sum_{c^{\prime} \mid \nu_{c c^{\prime}}=1} \sum_{l \in \mathbb{L}}\left[\left(\bar{\tau}_{l r, \mathrm{src}}+\hat{\tau}_{l r, \mathrm{src}} d_{c c^{\prime}}\right) \mathscr{Q}_{l c c^{\prime} h d t y}\right] \\
\forall r \in \mathbb{R}, c \in \mathbb{C}, h \in \mathbb{H}, d \in \mathbb{D}, t \in \mathbb{T}, y \in \mathbb{Y}
\end{array}
$$




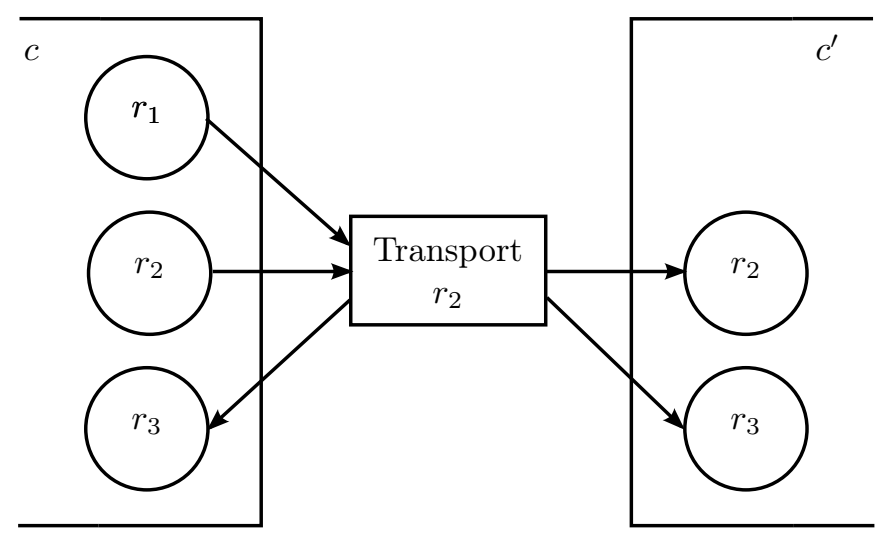

Figure 6: An example task to transport resource $r_{2}$.

The two terms on the right-hand side of the equation are the flows of resource in and out of cell $c$, respectively. If connections are allowed between all cells then this may result in a prohibitively large model (i.e. the number of variables, $\mathscr{Q}_{l c c^{\prime} h d t y}$, is proportional to the square of the number of cells). For computational efficiency, transport between cells is limited to adjacent cells - also known as the Moore neighbourhood [52]. Figure 7 shows a comparison of the possible transport connections between cell-to-cell transport and Moore neighbourhood transport. In the latter, transport over long distances is achieved by making several neighbour-to-neighbour transfers along the route between the source and destination cells. The binary parameter $\nu_{c c^{\prime}}$ indicates the feasible transport connections, i.e. it has a value of 1 if cell $c$ is adjacent to cell $c^{\prime}, 0$ otherwise. $\mathbb{L}$ is the set of transport technologies, an element of which is represented by the index $l$.

The parameters $\bar{\tau}_{l r f}$ and $\hat{\tau}_{l r f}$ are the conversion factors that account for losses in transporting resource $r$; the former is distance-independent whereas the latter depends on distance. $\mathscr{Q}_{l c c^{\prime} h d t y}$ is a positive variable representing the rate of transport via mode $l$ from cell $c$ to cell $c^{\prime}$. The distance between cells $c$ and $c^{\prime}$ is calculated as $d_{c c^{\prime}}=\sqrt{\left(x_{c}-x_{c^{\prime}}\right)^{2}+\left(y_{c}-y_{c^{\prime}}\right)^{2}}$, where $x_{c}$ and $y_{c}$ are the $\mathrm{x}$ - and y-coordinates of the centre of cell $c$. 


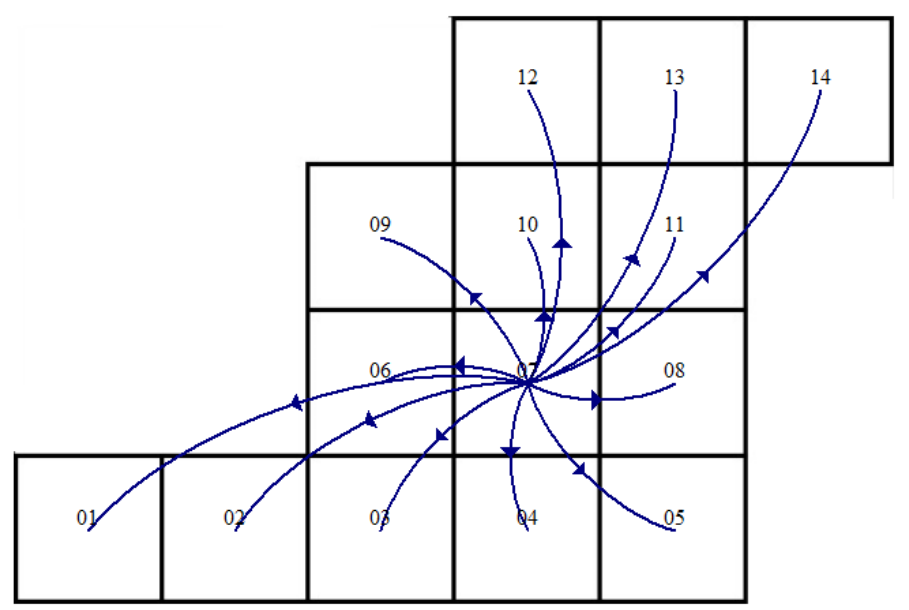

(a)

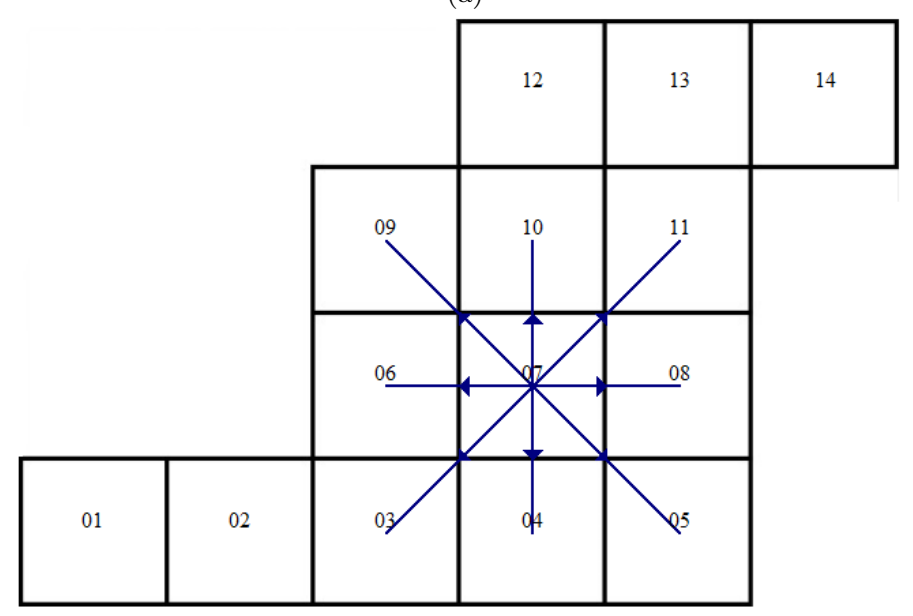

(b)

Figure 7: Comparison of (a) cell-to-cell transport and (b) Moore neighbourhood transport from cell 7. The arrows indicate all possible links from cell 7.

Infrastructures, such as roads, railways and pipelines, connect different cells and allow resources to be transported to different locations. The index $b$ represents an element in the set of transport infrastructures $\mathbb{B}$. The binary variable, $B_{b c c^{\prime}}$, determines whether infrastructure $b$ is present from cell $c$ to cell $c^{\prime}: B_{b c c^{\prime}}=1$ if infrastructure $b$ exists between cells $c$ and $c^{\prime} ; 0$ otherwise. The binary parameter, $L B_{l b}$, indicates if transport technology $l$ is supported by infrastructure $b: L B_{l b}=1$ if transport technology $l$ can use infrastructure $b ; 0$ otherwise.

The transport rate is constrained by $q_{l}^{\max }$, which denotes the maximum transfer rate via transport mode $l$, and whether an infrastructure exists between cells $c$ and $c^{\prime}$ :

$$
\mathscr{Q}_{l c c^{\prime} h d t y} \leq\left.\sum_{b \in \mathbb{B}} q_{l}^{\max } B_{b c c^{\prime}}\right|_{L B_{l b}=1 \wedge \nu_{c c^{\prime}}=1} \quad \forall l \in \mathbb{L} ; c, c^{\prime} \in \mathbb{C} ; h \in \mathbb{H}, d \in \mathbb{D}, t \in \mathbb{T}, y \in \mathbb{Y}
$$

The transport rate is also limited by $b_{b}^{\max }$, which is the maximum capacity of infrastructure $b$, as given by 
constraint 39. An infrastructure $b$ may be shared by different transport technologies, hence the summation over $l$.

$$
\sum_{l \in \mathbb{L}} \mathscr{Q}_{l c c^{\prime} h d t y} L B_{l b} \leq b_{b}^{\max } B_{b c c^{\prime}} \quad \forall b \in \mathbb{B} ; c, c^{\prime} \in \mathbb{C} ; h \in \mathbb{H}, d \in \mathbb{D}, t \in \mathbb{T}, y \in \mathbb{Y}
$$

The model also considers three types of infrastructure: bidirectional (e.g. electricity and gas networks); independent bidirectional, which only goes in one direction but can be built in both directions at twice the cost (e.g. roads, railways); and unidirectional, which may only be built in one direction (e.g. $\mathrm{CO}_{2}$ pipelines going toward a location where it is sequestered). The parameter $\beta_{b}$ indicates the directionality of infrastructure $b: \beta_{b}=1$ if infrastructure $b$ is bidirectional; $\beta_{b}=0$ if independent bidirectional; and $\beta_{b}=-1$ if unidirectional.

For bidirectional infrastructures:

$$
B_{b c c^{\prime}}=B_{b c^{\prime} c} \quad \forall b \in \mathbb{B} \mid \beta_{b}=1, c, c^{\prime} \in \mathbb{C}
$$

For unidirectional infrastructures:

$$
B_{b c c^{\prime}}+B_{b c^{\prime} c} \leq 1 \quad \forall b \in \mathbb{B} \mid \beta_{b}=-1, c, c^{\prime} \in \mathbb{C}
$$

\subsection{Resource import and export}

The rates of import and export in cell $c$ are limited by $m_{r c}^{\max }$ and $x_{r c}^{\max }$, which are the maximum rates of import and export, respectively.

$$
\begin{aligned}
M_{r c h d t y} \leq m_{r c}^{\max } & \forall r \in \mathbb{R}, c \in \mathbb{C}, h \in \mathbb{H}, d \in \mathbb{D}, t \in \mathbb{T}, y \in \mathbb{Y} \\
X_{\text {rchdty }} \leq x_{r c}^{\max } & \forall r \in \mathbb{R}, c \in \mathbb{C}, h \in \mathbb{H}, d \in \mathbb{D}, t \in \mathbb{T}, y \in \mathbb{Y}
\end{aligned}
$$

\subsection{Objective function}

All of the activities associated with the provision of energy, from the primary energy source to end-use, give rise to a number of financial and environmental impacts. For example, building and operating technologies for converting resource obviously incur capital and operating costs, along with other environmental impacts; building transport infrastructures and moving resources from one place to another incur a cost and also result in GHG emissions. These impacts can be expressed as a function of one or more decision variables in the problem, e.g. the amount of capacity of a technology installed, the rate of operation of technology, the rate of transport of a resource and so on. Each type of impact, $i$, is an element of the set of impacts $\mathbb{I}$. In the example problem, only cost is considered as an impact but it is relatively straightforward to include 
additional impacts (e.g. environmental impacts such as GHG emissions and social impacts such as number of potential jobs created).

The objective function, $Z$, is a weighted sum of all of the impacts of the whole-system:

$$
Z=\sum_{i} \omega_{i}\left[\left(\mathscr{I}_{i}^{u}+\mathscr{I}_{i}^{p}+\mathscr{I}_{i}^{s}+\mathscr{I}_{i}^{q}+\mathscr{I}_{i}^{m}+\mathscr{I}_{i}^{x}\right)+\left(\mathscr{I}_{i}^{P}+\mathscr{I}_{i}^{S}+\mathscr{I}_{i}^{Q}\right) / \gamma_{i}\right]
$$

where $\omega_{i}$ is a weighting parameter that allows different objective functions to be defined (e.g. minimise cost, minimise GHG emissions or a combination of these). The terms in the first pair of parentheses are the impacts due to operation whereas those in the second pair are due to capital expenditures. The individual impacts, $\mathscr{I}$, are given by equations 45 to 53 . The factor $\gamma_{i}$ converts the capital impact to the same time basis as the operating impact (e.g. if the time horizon is one year, then $\gamma_{i}$ are capital charge factors; if the time horizon is as long as the lifetime of the assets, then $\gamma_{i}=1$ because the operating costs are for the whole time horizon).

The total impact of utilising locally-available resources or purchasing resources from the "grid":

$$
\mathscr{I}_{i}^{u}=\sum_{r \in \mathbb{R}} \sum_{c \in \mathbb{C}} \sum_{h \in \mathbb{H}} \sum_{d \in \mathbb{D}} \sum_{t \in \mathbb{T}} \sum_{y \in \mathbb{Y}} R P I_{\text {richdty }} U_{\text {rchdty }} n_{h}^{h d} n_{d}^{d w} n_{t}^{w t} n_{y}^{y y} \quad \forall i
$$

where $R P I_{\text {richdty }}$ is the impact due to utilisation of one unit of resource $r$ in cell $c$ at time $h, d, t$ and $y$ (e.g. purchase price of resource $r$ from the "grid" or emissions associated with obtaining it locally).

The total impact of operating the conversion technologies:

$$
\mathscr{I}_{i}^{p}=\sum_{p \in \mathbb{P}} \sum_{c \in \mathbb{C}} \sum_{h \in \mathbb{H}} \sum_{d \in \mathbb{D}} \sum_{t \in \mathbb{T}} \sum_{y \in \mathbb{Y}} T O I_{p i} \mathscr{P}_{p c h d t y} n_{h}^{h d} n_{d}^{d w} n_{t}^{w t} n_{y}^{y y} \quad \forall i
$$
costs).

The total impact of operating the storage technologies:

$$
\mathscr{I}_{i}^{s}=\sum_{s \in \mathbb{S}} \sum_{c \in \mathbb{C}} \sum_{h \in \mathbb{H}} \sum_{d \in \mathbb{D}} \sum_{t \in \mathbb{T}} \sum_{y \in \mathbb{Y}}\left(S P I_{s i} \mathscr{S}_{\text {schdty }}^{\text {put }}+S H I_{s i} \mathscr{S}_{\text {schdty }}^{\text {hold }}+S G I_{s i} \mathscr{S}_{\text {schdty }}^{\text {get }}\right) n_{h}^{h d} n_{d}^{d w} n_{t}^{w t} n_{y}^{y y} \quad \forall i
$$

where $S P I_{s i}, S H I_{s i}$ and $S G I_{s i}$ are the impacts per unit rate of charging, maintaining and discharging a storage technology $s$, respectively.

The total cost of transporting the resources: 


$$
\mathscr{I}_{i}^{q}=\sum_{l \in \mathbb{L}} \sum_{c \in \mathbb{C}} \sum_{c^{\prime} \in \mathbb{C}} \sum_{h \in \mathbb{H}} \sum_{d \in \mathbb{D}} \sum_{t \in \mathbb{T}} \sum_{y \in \mathbb{Y}}\left(\bar{T} I_{l i}+\hat{T} I_{l i} d_{c c^{\prime}}\right) \mathscr{Q}_{l c c^{\prime} h d t y} n_{h}^{h d} n_{d}^{d w} n_{t}^{w t} n_{y}^{y y} \quad \forall i
$$

where $\bar{T} I_{l i}$ and $\hat{T} I_{l i}$ are the impacts due to one unit of operation of transport technology $l$; the former is distance-independent while the latter is per unit distance.

The total impact of importing resources:

$$
\mathscr{I}_{i}^{m}=\sum_{r \in \mathbb{R}} \sum_{c \in \mathbb{C}} \sum_{h \in \mathbb{H}} \sum_{d \in \mathbb{D}} \sum_{t \in \mathbb{T}} \sum_{y \in \mathbb{Y}} M I_{r i} M_{r c h d t y} n_{h}^{h d} n_{d}^{d w} n_{t}^{w t} n_{y}^{y y} \quad \forall i
$$

where $M I_{r i}$ is the impact of importing one unit of resource $r$.

The total impact of exporting resources:

$$
\mathscr{I}_{i}^{x}=\sum_{r \in \mathbb{R}} \sum_{c \in \mathbb{C}} \sum_{h \in \mathbb{H}} \sum_{d \in \mathbb{D}} \sum_{t \in \mathbb{T}} \sum_{y \in \mathbb{Y}} X I_{r i} X_{r c h d t y} n_{h}^{h d} n_{d}^{d w} n_{t}^{w t} n_{y}^{y y} \quad \forall i
$$

where $X I_{r i}$ is the impact of exporting one unit of resource $r$.

The total capital impact of building the conversion technologies, storage facilities and transport infrastructures are given by equations 51 to 53 .

$$
\begin{aligned}
\mathscr{I}_{i}^{P} & =\sum_{p \in \mathbb{P}} \sum_{c \in \mathbb{C}} T C I_{p i} N_{p c}^{P} \quad \forall i \\
\mathscr{I}_{i}{ }^{S} & =\sum_{s \in \mathbb{S}} \sum_{c \in \mathbb{C}} S C I_{s i} N_{s c}^{S} \quad \forall i \\
\mathscr{I}_{i}{ }^{Q} & =\sum_{b \in \mathbb{B}} \sum_{c \in \mathbb{C}} \sum_{c^{\prime} \in \mathbb{C}} B I_{b} B_{b c c^{\prime}} d_{c c^{\prime}}\left(1-\left.0.5\right|_{\beta_{b}=1}\right) \quad \forall i
\end{aligned}
$$

where $T C I_{p i}, S C I_{s i}$ and $B I_{b}$ are the capital impacts of building a single conversion technology $p$, a single storage facility $s$, and 1 unit (e.g. $1 \mathrm{~km}$ ) of transport infrastructure $b$, respectively. The term $\left.0.5\right|_{\beta_{b}=1}$ only appears for bidirectional infrastructures, where $\beta_{b}=1$, so that although $B_{b c c^{\prime}}=B_{b c^{\prime} c}$ (see constraint 40 ) the cost of only a single connection is incurred. If $\beta_{b} \neq 1$, then this term disappears and connections in each direction are costed independently.

\section{Decomposition method}

650

One of the bottlenecks to solving large-scale spatio-temporal models is the very large number of binary variables associated with the transport infrastructure combined with the integer variables required to model 
the existence of conversion and storage technologies. This results in a very large search tree for the branchand-bound algorithm. If the LP problem that must be solved at each node is also very large due to a high temporal resolution in the model (i.e. $|\mathbb{H}| \times|\mathbb{D}| \times|\mathbb{T}| \times|\mathbb{Y}|$ is large), then the solution time can be restrictively high. In these cases, some form of decomposition will be required to obtain solutions in a reasonable time.

The decomposition approach applied in this paper is shown in Figure 8. The first stage is to reduce the temporal resolution to a manageable level while maintaining a full design formulation. Thus, the time horizon is limited to a single day (for each yearly interval) and a worst-case scenario is chosen so that the design will be conservative. In stage 2, the full temporal resolution (with the non-uniform hierarchical time discretisation) is restored and the transport infrastructure is fixed from stage 1; only the conversion and storage technologies are designed again, along with the operation of the transport network and the conversion and storage technologies. In the first iteration, the result of stage 2 is passed to stage 3 , where the conversion and storage technologies are now fixed and the transport infrastructure is re-designed, again using the full temporal resolution and also determining all of the operational variables. If the objective function has improved in stage 3, then stage 2 is performed again with the new transport infrastructure design from stage 3 ; otherwise the algorithm terminates and the solution from stage 3 is reported. Stages 2 and 3 are repeated until one of them does not return an improved objective function and the solution of this last stage is reported.

Note that this decomposition method is based on solving a model that has already been simplified by applying the non-uniform hierarchical time discretisation. Without this simplification, stage 1 is just the full problem with 8760 time intervals per year, which will almost certainly be intractable. Stages 2 and 3 would also contain contiguous-time formulations but with a fixed network (stage 2) or a fixed set of conversion and storage technologies (stage 3). However, these two stages are also likely to be intractable (each being more complex than the simple problem described in Section 6). 


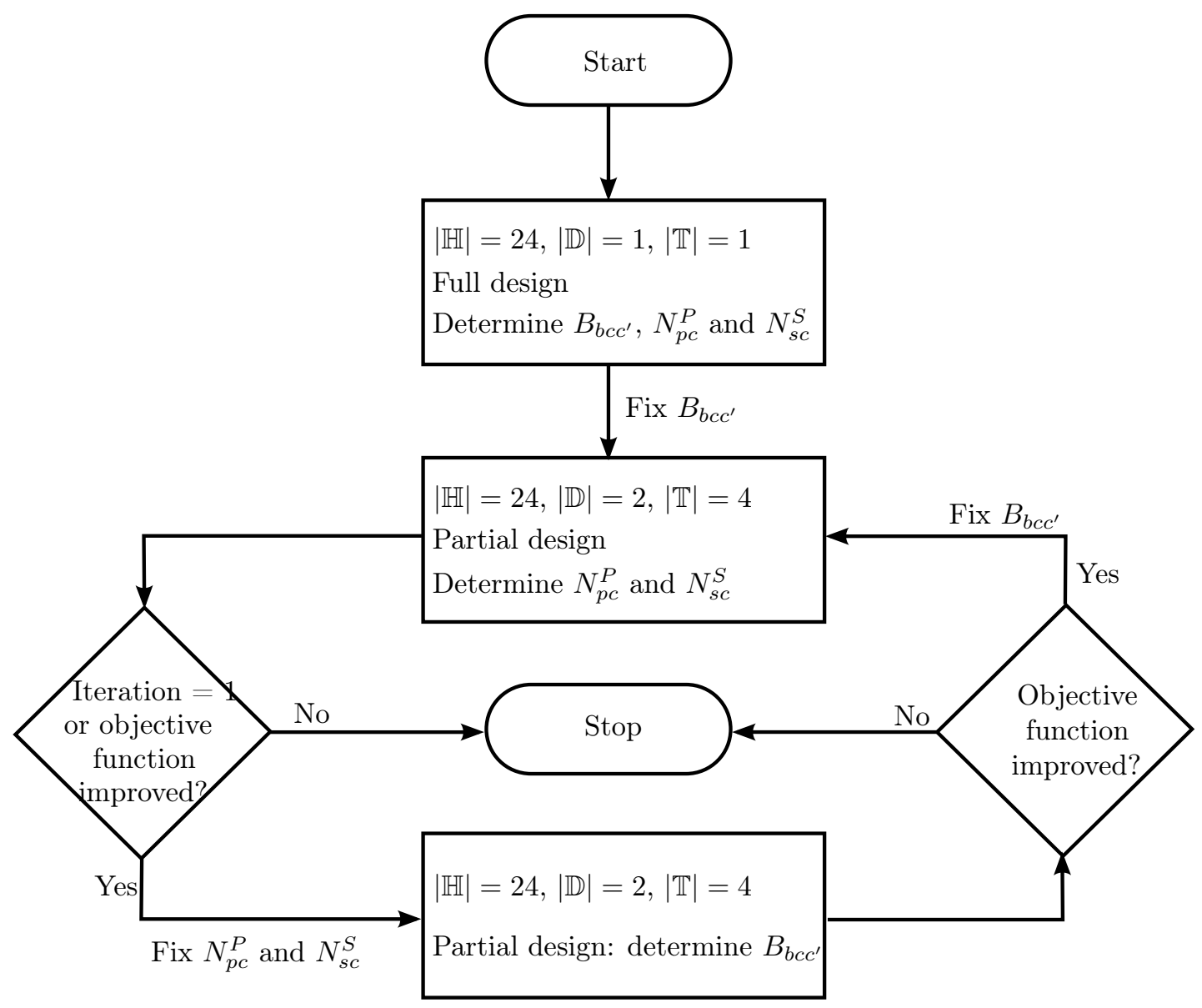

Figure 8: Flowchart of the decomposition algorithm

\section{Illustrative example}

In this section, the model presented in Section 8 is used to solve the hydrogen network design problem outlined in Section 4. Both the non-uniform hierarchical time discretisation and the decomposition method were applied. Without the former, the model would be intractable, as was shown in Section 6 for a much simpler model, and without the decomposition approach a solution was not obtained after two weeks of computation. It was therefore necessary to apply both methods as described below.

The data in Figure 2(b) were analysed to establish if there were any repeated patterns. Both the demand and wind availablity depend on the season. The demand also shows a distinct difference between week days and weekends. Therefore, four seasons and 2 day types were chosen. For the hourly interval, it was necessary to use 24 1-hour periods because the wind data were too variable: there is no fixed time during the day when all profiles are roughly constant, which would be necessary in order to apply a non-uniform hourly discretisation. One should select representative profiles for each combination of day type and season. It is important not to average the profiles in each day type because this would smooth out the variability; doing so would result in underestimating the capacity of the storage, production and transport technologies. In this case study, the most variable wind profiles for each day type and season were selected in order to obtain 
a conservative design. The time discretisation chosen is summarised below and the resulting demand profiles, wind availability profiles and electricity prices (it was assumed that the windfarms were owned by private investors and that any electricity taken from them, in order to generate hydrogen, has to be purchased) are shown in Figure 9.

- 4 different seasons in a year: $|\mathbb{T}|=4 ; \mathbb{T}=\{$ spring, summer, autumn, winter $\} ; n_{t}^{w t}=13 \forall t$

695

- 2 different day types in a week: $|\mathbb{D}|=2 ; \mathbb{D}=\{$ weekday (WD), weekend (WE) $\} ; n_{\mathrm{WD}}^{d w}=5, n_{\mathrm{WE}}^{d w}=2$

- 24 hours in a day: $|\mathbb{H}|=24 ; \mathbb{H}=\{1,2, \ldots, 24\} ; n_{h}^{h d}=1 \forall h$ 


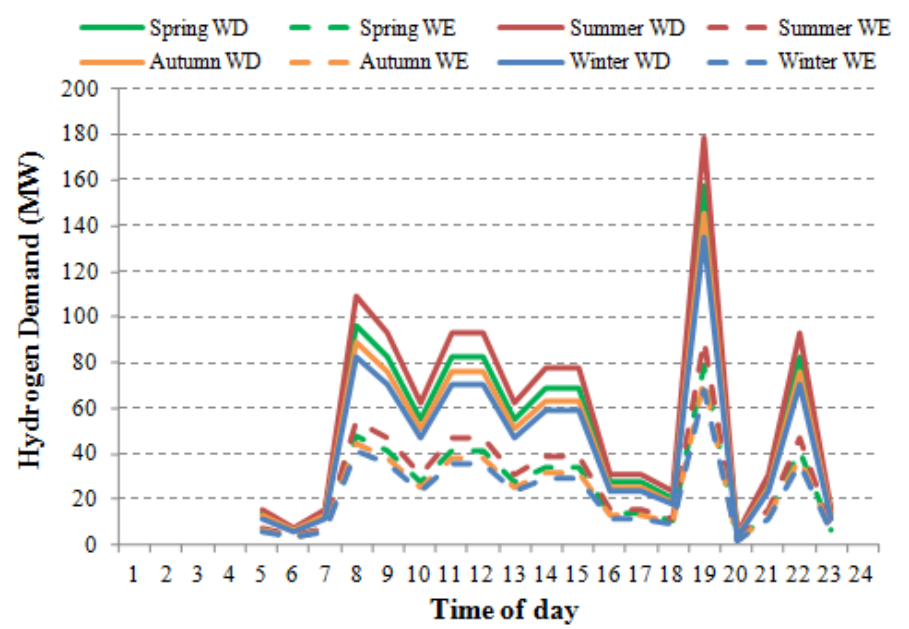

(a)

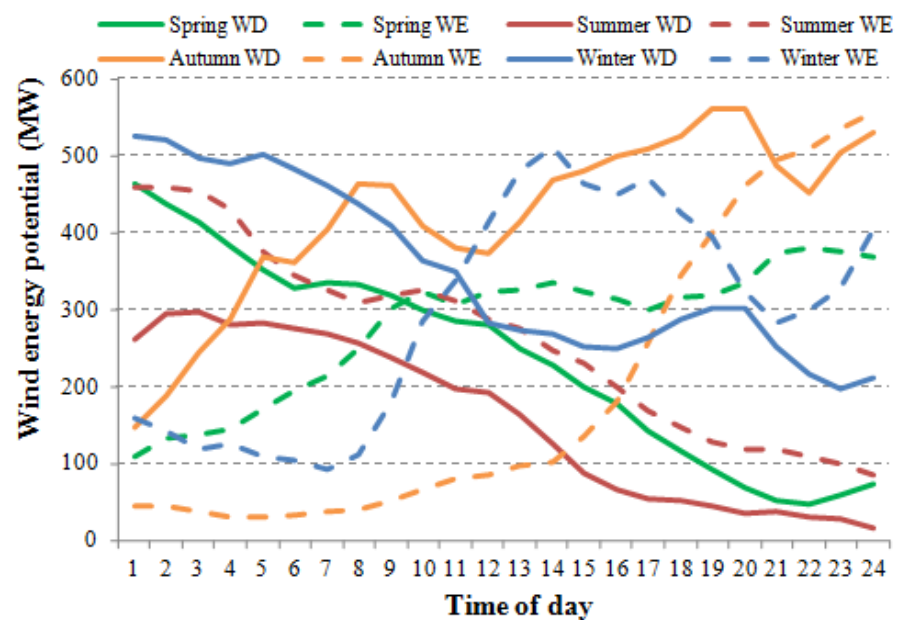

(b)

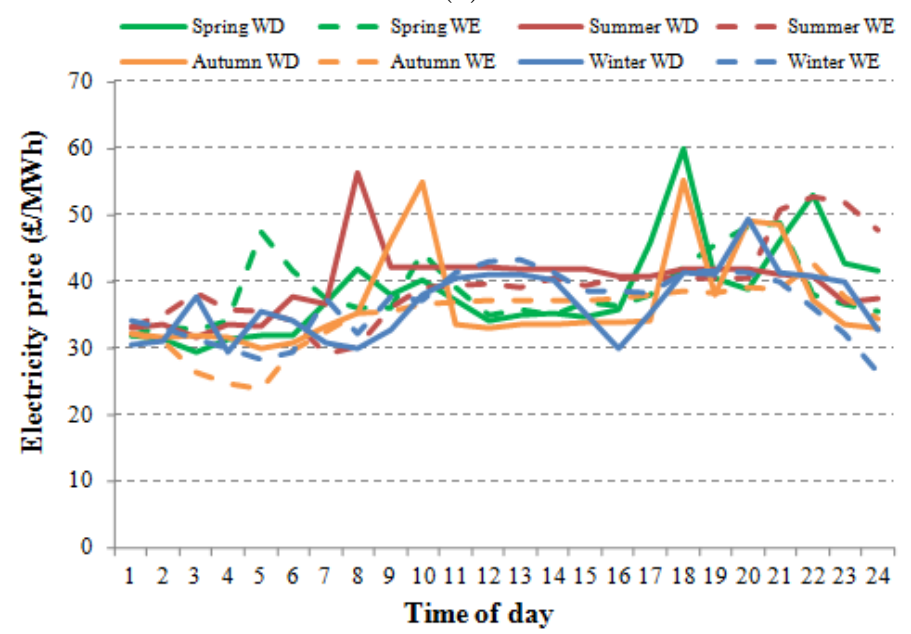

(c)

Figure 9: Temporal data applying a discretisation of 4 different seasons, 2 day types in a week and 24 hours in a day: (a) transport demand in cell 7; (b) wind energy potential; and (c) electricity prices. 
The characteristics of the conversion technologies are summarised in Table 2. The maximum capacity of a single technology was considered to be $50 \mathrm{t}$ /day of hydrogen. If one unit of a technology is not enough to meet the demand then more than one technology can be used (as determined by the integer variable $N_{p c}^{P}$ in the model). The data in Table 2 were estimated from the reports by the National Renewable Energy Laboratory [53, 54] and Amos [51], the paper of Almansoori and Shah [24] and an online presentation by the U.S. Department of Energy [55]. Table 3 gives the conversion factors that were used in this example. Negative values indicate consumption and positive values mean production, e.g. for fuel cell, $0.6 \mathrm{MWh}$ of electricity is produced for every $1 \mathrm{MWh}$ of $\mathrm{CGH}_{2}$ consumed.

Table 2: Characteristics of conversion technologies

\begin{tabular}{lrrrr}
\hline & Electrolysis & Fuel cell & Liquefier & Regasifier \\
\hline Max production rate of a single facility [MWh/h] & 69.38 & 69.38 & 69.38 & 69.38 \\
Capital cost of a single facility [M£] & 31.56 & 87.75 & 32.25 & 0.54 \\
Unit operating cost [£/MWh] & 7.21 & 6.60 & 11.26 & 0.10 \\
\hline
\end{tabular}

Table 3: Conversion factor of resource $r$ in conversion technology $p\left(\alpha_{r p}\right)$

\begin{tabular}{lrrr}
\hline Resource $r$ & Electricity & $\mathrm{CGH}_{2}$ & $\mathrm{LH}_{2}$ \\
\cline { 1 - 3 } Conversion technology $p$ & & & \\
\cline { 1 - 4 } Electrolysis & -1 & 0.666 & \\
Fuel cell & 0.6 & -1 & \\
Liquefier & & -1 & 0.95 \\
Regasifier & & 0.99 & -1 \\
\hline
\end{tabular}

Four different technologies for storing hydrogen were considered: underground, compressed gas, liquid and metal hydride. The properties of these storage technologies are given in Table 4 and are based on the data presented in the works of Amos [51], Thistlethwaite et al. [12] and Almansoori and Shah [24]. For underground storage, the capital cost comprises cavern and compressor costs. The cost of putting hydrogen into the storage includes cooling water and the electricity requirement of the compressor, which were calculated from the power and cooling requirements for compressing hydrogen from 1 atmosphere to $20 \mathrm{MPa}$. These also apply to compressed gaseous storage, but the capital cost includes the cost of the tank instead of the cavern. For liquid storage, the capital expense includes the cost of the insulated dewar and the operating cost includes the cooling requirement. Note that the cost of liquid storage does not include the cost of liquefying hydrogen as the liquifier is considered as a separate conversion technology. For the liquefier, the capital and operating costs are given in Table 2. The power and cooling requirements for the liquefier were assumed to be $10 \mathrm{kWh}$ electricity and $626 \mathrm{~L}$ water per kilogram of hydrogen liquefied. For metal hydride, cooling water is needed to store the hydrogen in its hydride form and heat (e.g. steam) is required to release the hydrogen from the hydride. The capital cost includes the hydride material and an integrated heat exchanger and the operating cost includes the energy requirement for cooling and heating during adsorption and desorption. The conversion factors for the different storage tasks are shown in Table 5. For liquid storage, a boil-off rate of $0.1 \%$ per day [51] was assumed, resulting in an efficiency of $99.9 \%$. 
Table 4: Characteristics of storage technologies

\begin{tabular}{lrrrr}
\hline & Underground & Compressed gas & Liquid & Metal hydride \\
\hline Maximum storage capacity of a single facility [GWh] & 30 & 3.63 & 3.63 & $7.56 \times 10^{-3}$ \\
Capital cost of a single facility [M£] & 4.78 & 2.75 & 2.78 & 0.50 \\
Unit cost for putting $\mathrm{H}_{2}$ into storage [£/MWh] & 2.16 & 2.16 & 0 & 0.75 \\
Unit cost for maintaining $\mathrm{H}_{2}$ in storage [£/MWh] & 0 & 0 & 0 & 0 \\
Unit cost for withdrawing $\mathrm{H}_{2}$ from storage [£/MWh] & 0 & 0 & 0 & 1.59 \\
\hline
\end{tabular}

\begin{tabular}{lccc}
\cline { 2 - 2 } & Flow direction & Source & Destination \\
\cline { 1 - 1 } Storage & Resource & & \\
\hline Underground & $\mathrm{CGH}_{2}$ & -1 & 1 \\
Compressed gas & $\mathrm{CGH}_{2}$ & -1 & 1 \\
Liquid & $\mathrm{LH}_{2}$ & -1 & 1 \\
Metal hydride & $\mathrm{CGH}_{2}$ & -1 & 1 \\
\hline
\end{tabular}

(a)

\begin{tabular}{|c|c|c|c|}
\hline & Flow direction & Source & Destination \\
\hline Storage & Resource & & \\
\hline Underground & $\mathrm{CGH}_{2}$ & 1 & 0 \\
\hline Compressed gas & $\mathrm{CGH}_{2}$ & 1 & 0 \\
\hline Liquid & $\mathrm{LH}_{2}$ & 0.999 & 0 \\
\hline Metal hydride & $\mathrm{CGH}_{2}$ & 1 & 0 \\
\hline
\end{tabular}

(b)

\begin{tabular}{llrr} 
& Flow direction & Source & Destination \\
\cline { 2 - 2 } Storage & Resource & & \\
\hline Underground & $\mathrm{CGH}_{2}$ & -1 & 1 \\
Compressed gas & $\mathrm{CGH}_{2}$ & -1 & 1 \\
Liquid & $\mathrm{LH}_{2}$ & -1 & 1 \\
Metal hydride & $\mathrm{CGH}_{2}$ & -1 & 1 \\
\hline
\end{tabular}

(c)

Table 5: Conversion factors for the different stages of storage: (a) "put" task; (b) "hold" task; and (c) "get" task.

The transport technologies considered include pipeline, truck and rail. For gaseous hydrogen transport by pipeline, a large fraction of the capital cost is for installation so the cost was based on that for natural gas. Amos [51] estimated the cost of pipeline installation and construction to be $£ 0.37 \mathrm{M} / \mathrm{km}$. The operating cost includes the power requirement of the compressor, which Amos [51] estimated to be $£ 0.094 / \mathrm{MWh} / \mathrm{km}$. The cost of a new rail infrastructure is $£ 11 \mathrm{M} / \mathrm{km}$, while for roads the cost is $£ 1.5 \mathrm{M} / \mathrm{km}[56]$. The cost of one tanker truck, which includes the tank, cab and undercarriage, is approximately £0.26M [24]. The cost of one railway tank car, which includes tank and undercarriage, is also approximately £0.26M [24]. For liquid hydrogen, the unit operating cost is $£ 1.26 / \mathrm{MWh} / \mathrm{km}$ which includes the fuel, driver and maintenance charges [51]. This is higher for compressed gas transport by truck, which is $£ 2.03 / \mathrm{MWh} / \mathrm{km}$. Finally, the operating cost of transporting hydrogen by rail is a flat rate (i.e. independent of distance): $263.06 / \mathrm{MWh}$ for compressed gas and £101.53/MWh for liquid [51]. The conversion factors for transport processes are given in Table 6. A boil-off rate of $0.3 \%$ day [51] was used for liquid hydrogen transport resulting in an efficiency of $99.9875 \%$. Here, transport losses are assumed to be independent of distance, hence only the conversion factor $\bar{\tau}_{l r f}$ was specified. In cases where transport loss depends on distance, e.g. electricity transmission, 
then the conversion factor $\hat{\tau}_{l r f}$ needs to be provided (see Section 8.4).

Table 6: Distance-independent conversion factor when transporting resource $r$ by process $l\left(\bar{\tau}_{l r f}\right)$. The source is cell $c$ and the destination is cell $c^{\prime}$.

\begin{tabular}{llrr}
\cline { 2 - 2 } & Flow direction & Source & Destination \\
\cline { 1 - 1 } Transport & Resource & & 1 \\
Pipeline & $\mathrm{CGH}_{2}$ & -1 & -1 \\
Truck & $\mathrm{LH}_{2}$ & -1 & 0.999875 \\
Truck & $\mathrm{CGH}_{2}$ & -1 & 0.999875 \\
Rail & $\mathrm{LH}_{2}$ & -1 & 1 \\
Rail & $\mathrm{CGH}_{2}$ & & \\
\hline
\end{tabular}

For simplicity, only the economic impact was considered in this example. However, when data are available for other impacts, such as GHG emissions, it is easy to include them in the formulation by adding them as elements to the set of impacts $\mathbb{I}$ and providing values for relevant parameters such as $R P I_{\text {richdty }}, \hat{T} I_{l i}$,

given in Table 8. Since there are no significant improvement in the objective function value (i.e. the change is within the specified tolerance of $0.1 \%$ ) after the third iteration, the solution from this stage was accepted.

Table 7: Summary of computational results of the three stages of the decomposition method illustrated in Figure 8.

\begin{tabular}{rrrrrr}
\hline Iteration & Stage & No. of variables & No. of constraints & CPU time (s) & Objective function value \\
\hline 1 & 1 & 19,191 (700 integer) & 57,985 & 60,844 & 174.172 \\
2 & 2 & 147,289 (112 integer) & 384,489 & 1,266 & 146.648 \\
3 & 3 & 147,767 (588 integer) & 461,857 & 81,596 & 146.647 \\
\hline
\end{tabular}

Figure 10 shows the resulting network structure, i.e. the number, location and size of hydrogen production and storage facilities and the type of transport infrastructure established between cells. Two electrolyser 
units, each with a capacity of $69.4 \mathrm{MW}$, are installed near to the wind turbines to convert electricity to gaseous hydrogen, which is then transported by pipelines to satisfy the different demands of each cell. It can be seen that the network covers the shortest possible distance and that there are two separate pipeline networks. This is because the model does not evaluate the robustness of the network and therefore it is not possible, at this stage, to include constraints to ensure there is sufficient redundancy in the network. There are 5 compressed hydrogen gas storage units (a total capacity of $18.15 \mathrm{GWh}$ ) installed in different locations: 3 of these are located near the hydrogen-generating technologies and 2 are close to the cell with highest demand.

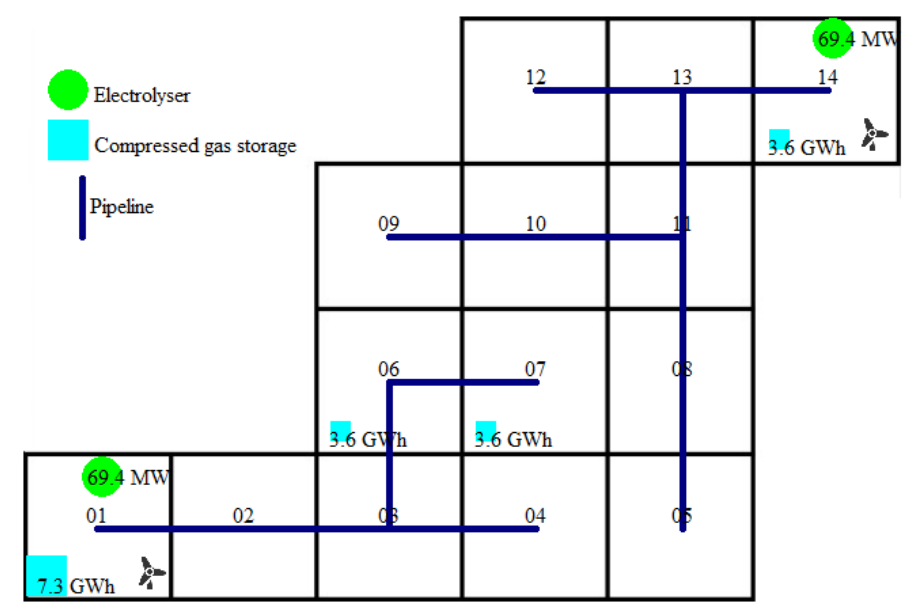

Figure 10: Optimal network structure: electrolysers are installed in the same cells as wind turbines; compressed gas storage units are located in the same cells as hydrogen-generating technologies and near the cells with highest demand; and pipeline networks are established to transport hydrogen between cells.

Figure 11 illustrates how storage can enable the integration of intermittent renewable sources into the energy system. Without storage, the problem is infeasible because there are points in time at which the available wind power is lower than the demand. With storage, however, only a fraction of the available wind energy is actually utilised as shown in Figures 11(a)-(b). Consequently, only one electrolyser for each wind farm is needed to satisfy the demand for hydrogen. The electrolysers run at full capacity for most of the time, which is most efficient. 

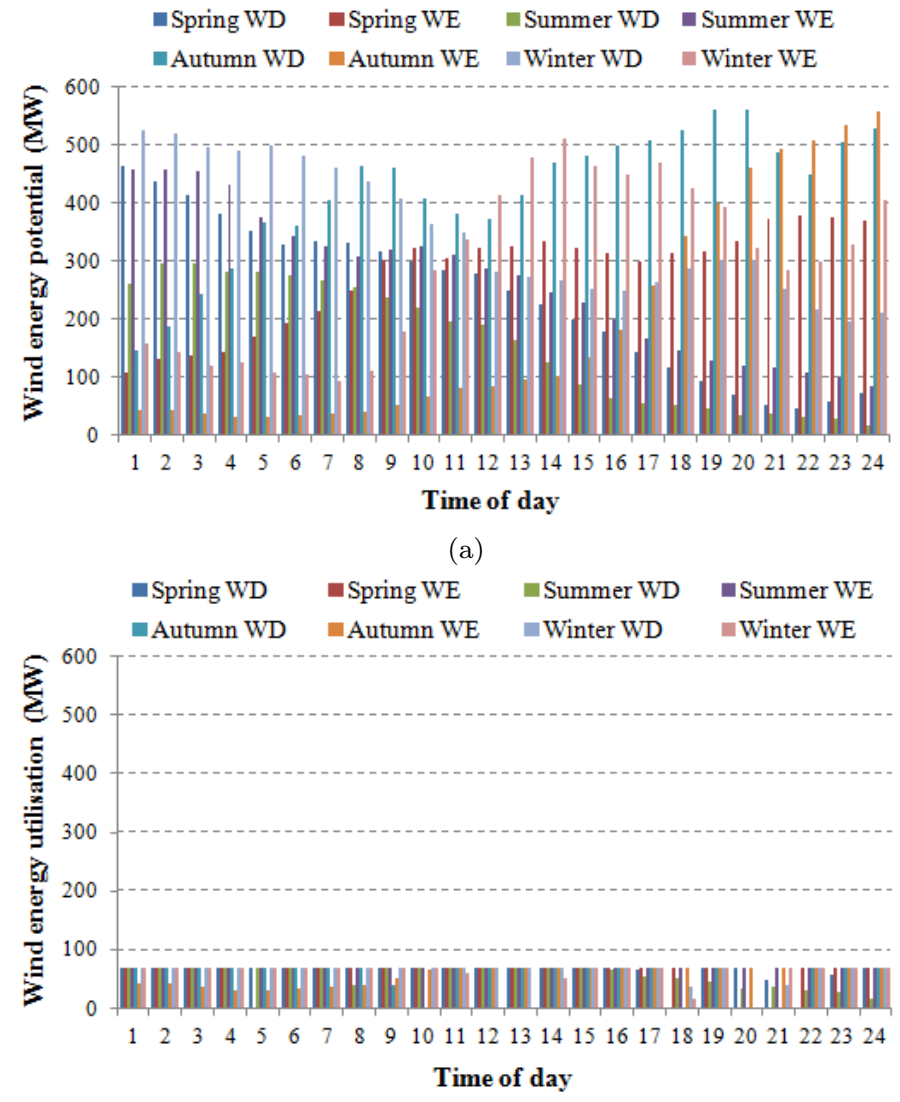

(b)

Figure 11: (a) Wind potential and (b) utilisation in cell 1 for all time intervals. Without storage the problem is infeasible but with storage the demands for hydrogen can be met using a fraction of the available wind energy.

Figure 12(a) shows a snapshot of the operation of compressed gas storage installed at different locations in the island during a weekday in summer; Figure 12(b) shows the demand at different cells during the same time period for comparison. The storage technologies are being charged when the demand is low and discharged when the demand is high. Figures 13(a)-(c) show the transport flow rates of hydrogen at different times of a weekday in summer. These results confirm that storage plays a very important role in meeting the demand particularly at peak times. 


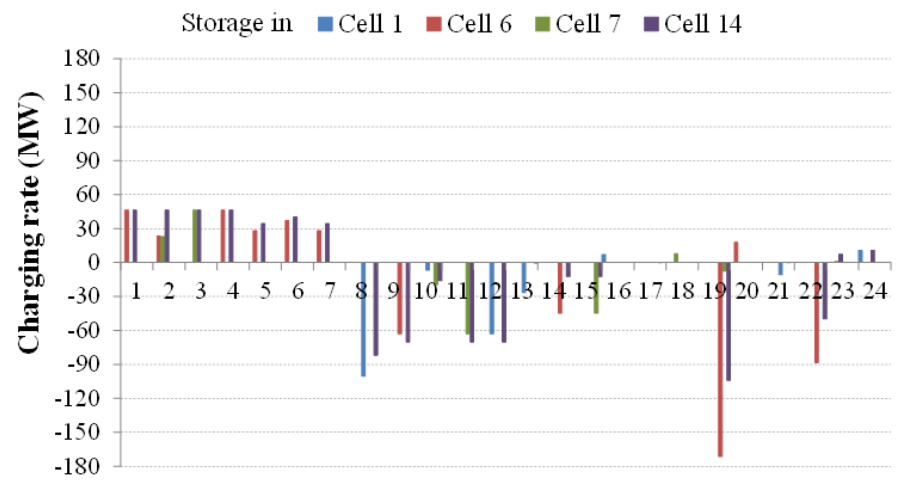

\section{Time of day}

(a)

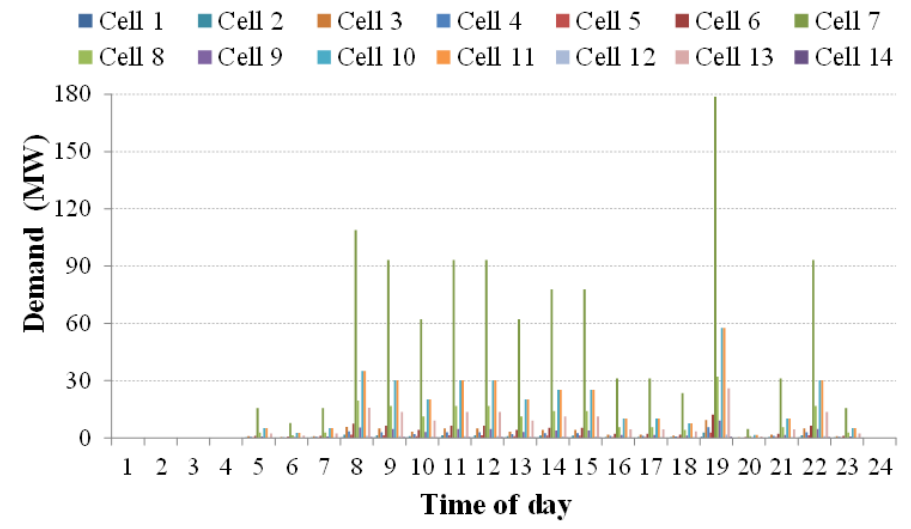

(b)

Figure 12: (a) A snapshot of the operation of compressed gas storage installed at different locations in the island during a weekday in summer and (b) the demands at different cells for comparison . 


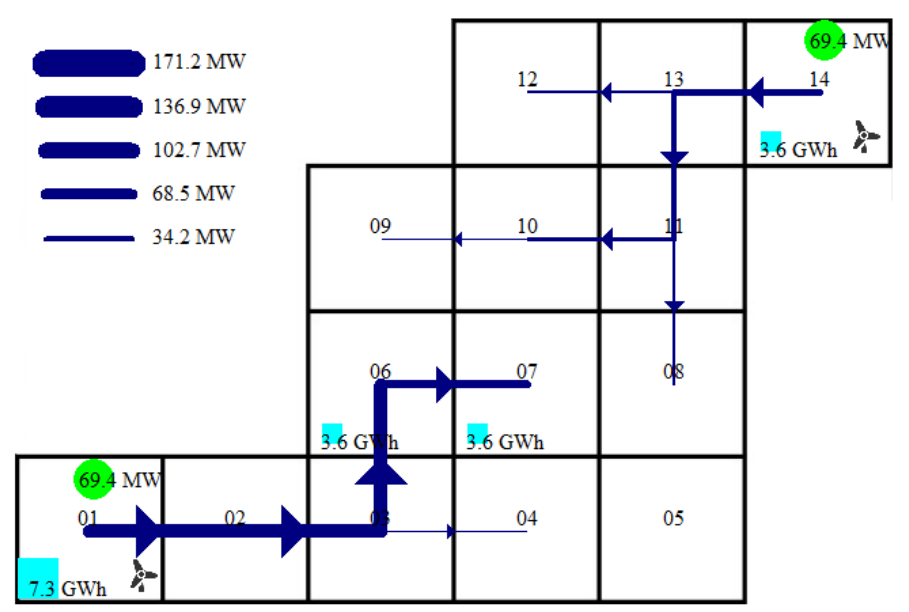

(a)

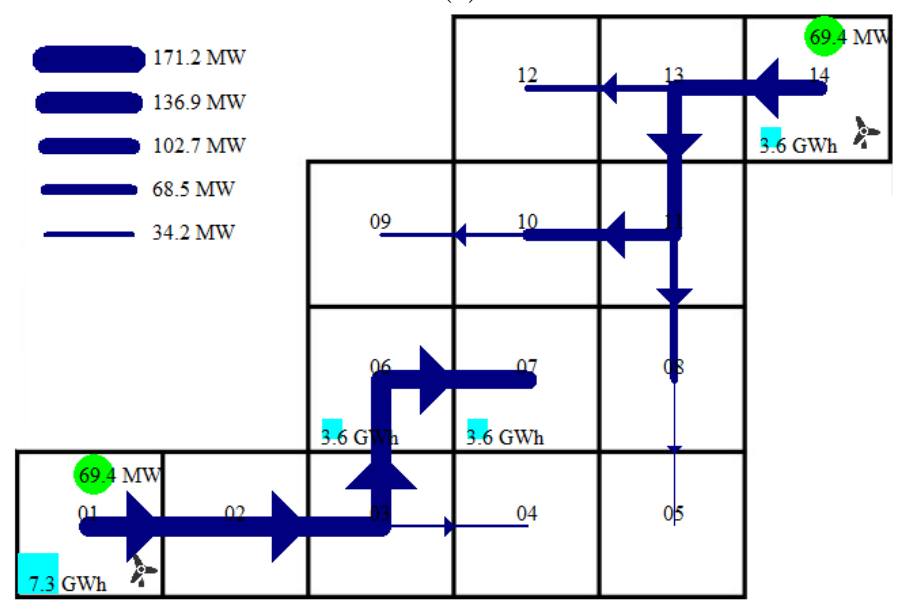

(b)

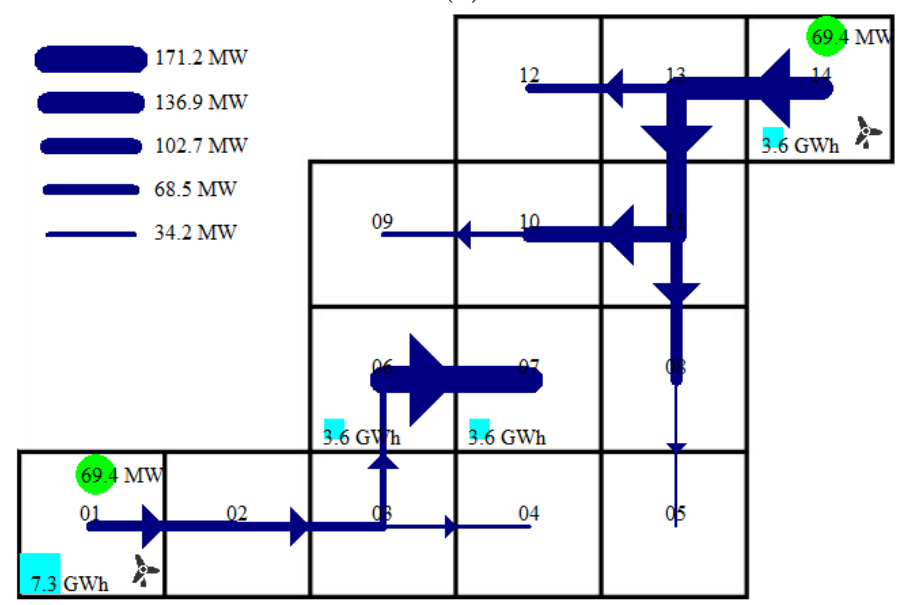

(c)

Figure 13: Snapshots of transport operation at different times of a weekday in summer: (a) 7:00; (b) 12:00; and (c) 19:00.

Figure 14 shows the inventory in all 5 storage facilities for a whole year, at the hourly level. In Figure 14(a), it can be seen that the storage in cell 1 (two facilities) is completely full throughout the whole of spring. 
Over summer the storage is consumed and is almost empty during autumn. Since there is a cyclic constraint on storage, the inventory is replenished over the winter months. One can also see the short-term variations during each week of each season (apart from spring, since the storage is full). Figures 14(b) and (c) show a very similar trend for cells 6 and 7, respectively. Both storage devices are nearly fully utilised in spring. Cell 6 is used much more for short-term storage than cell 7 (i.e. the change in inventory over a week is more pronounced), though both have very similar long-term storage - similar also to the storage in cell 1. Finally, the storage in cell 14 (Figure 14(d)) has a noticably different inventory profile: rather than staying low during autumn and replenishing over winter, the inventory is replenished in autumn to near full capacity and is roughly constant during winter.

The breakdown of costs for establishing a hydrogen network in the island is shown in Figure 15. The total cost is dominated by the large investment in pipeline infrastructure. The reason that the cavern, a lower-cost storage option per MWh of hydrogen, was not favoured is because establishing one in cell 9 results in a higher pipeline capital cost. Figure 16 shows the network structure when the cavern in cell 9 is used as storage: the storage cost (capital and operating) is only $2.08 £ \mathrm{M} / \mathrm{yr}$ (cheaper by $3.16 £ \mathrm{M} / \mathrm{yr}$ ) but the pipeline capital cost is $80.17 £ \mathrm{M} / \mathrm{yr}(6.17 £ \mathrm{M} / \mathrm{yr}$ more expensive)

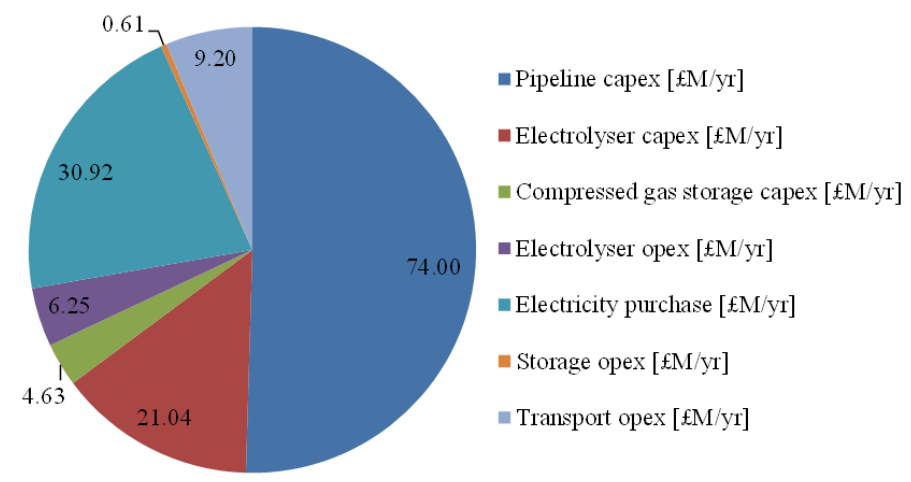

Figure 15: Breakdown of costs for establishing a hydrogen network in the island. 

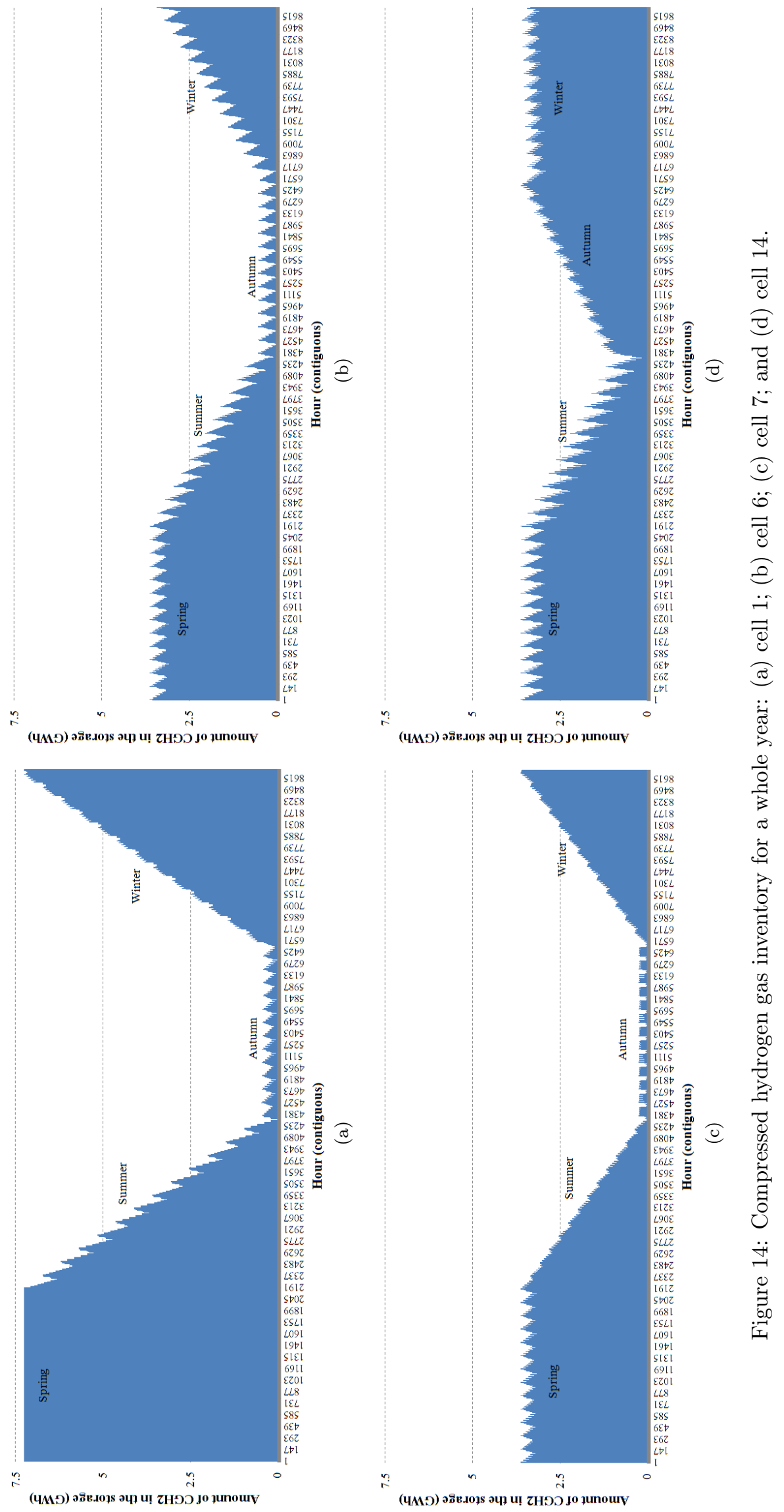


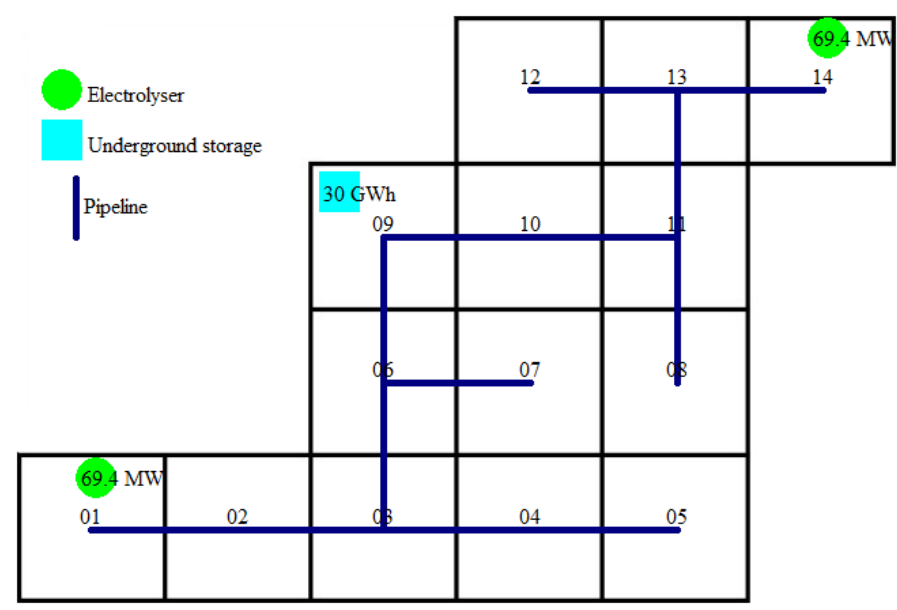

(a)

Figure 16: The network structure when the cavern in cell 9 is utilised. In this case, storage is $3.16 £ \mathrm{M} / \mathrm{yr}$ cheaper but the pipeline cost is $6.17 £ \mathrm{M} / \mathrm{yr}$ more expensive than in a network with a distributed compressed gas storage.

\section{Conclusions}

This paper presents a spatio-temporal mathematical model for the design and operation of multi-vector energy systems that allows detailed short-term operation to be considered along with long-term planning decisions. The model uses an RTN (Resource-Technology Network) representation of the energy pathways, which is also applied to transport and storage of resources. This allows one to account for losses and requirements for other resources when storing and transporting any resource - thus the interactions between the different energy networks are explicitly considered. The model determines the type, location, number and size of conversion and storage technologies along with their operation on an hourly level; transport infrastructures are also designed while optimising the rates of transport of various resources utilising them. All of this is done considering the short-term dynamics and a long-term planning horizon simultaneously, to optimise a linear combination of cost, emissions and so on.

To model storage accurately, hourly or shorter intervals are needed to capture its dynamics. Model tractability is the main challenge: as demonstrated in this paper, even a very simple MILP model that accounts for resource generation/conversion, storage and transport becomes intractable when considering a horizon of a season or longer at an hourly level. The non-uniform hierarchical time discretisation, along with appropriate constraints to link the different time periods, allows a much longer time horizon to be considered by exploiting periodicity in the data. The decomposition algorithm also helps to reduce the computational burden.

The example problem in this paper demonstrates how a time horizon of effectively one year with 1-hour intervals can be considered, which to the authors' knowledge is something that existing models in the literature, that attempted to optimise the size and location of energy storage, could not achieve. However, this was only possible by assuming that the system is periodic: that the hourly profile for each day type is the same, each week of the season is the same and each year in the yearly intervals is the same. If this is 
not the case in reality, then some information will inevitably be lost. It is difficult to determine exactly how much accuracy is lost by adopting this approach because it is currently not possible to solve this model with the full 8760 hours per year using the available solvers. How useful this approach will be clearly depends on the data but to mitigate some of the loss in accuracy, one should aim to use the highest time resolution that can be solved in a reasonable time and to use the most variable profiles as the representative daily profiles in the hope that the solution will be a conservative one.

The example problem also illustrates how storage can play an essential role in realising the full potential of intermittent renewables. For example, without storage the example problem is infeasible but with storage, only a small fraction of available primary resource is used and the generation technology operates effectively at a constant rate.

The presented formulation is intended to be general, i.e. none of the constraints are specific to a certain location, or set of resources, technologies and so on. It can be applied easily to any region of interest such as a city or a country by providing the necessary dataset. Similarly, extending the model to include other energy vectors will involve defining new resources and technologies and specifying their required characteristics (or parameters). Additional constraints may be added when adapting the model to a specific scenario, e.g. when applying to heat systems the transport of heat could be restricted to a certain distance or when considering a specific region of interest there are sites that may not be suitable for establishment of a particular technology. Extending the model to consider staged investments and retirements of old assets is relatively straightforward and will involve adding a number of constraints similar to those in the BVCM [22].

The set of constraints presented in this paper could form a base for future specific models that consider systems that exhibit significant spatial and temporal dependencies. For realistic problems, however, difficulty relating to the size of the resulting MILP model may yet be encountered. Therefore, the spatial and temporal resolutions that are going to be used in the model require careful consideration, bearing in mind the trade-off between level of detail and computational efficiency. Non-uniform hourly intervals can be used to reduce further the size of the problem without compromising the accuracy of the model and decomposition methods, such as the one presented in this paper, will also be useful for obtaining solutions within an acceptable time. For the hydrogen network, the model will be extended to include pipeline storage and hydrogen injection into the natural gas grid. The model will also be applied to heat systems to evaluate the role of thermal storage in low-carbon heat networks. Finally, since the pathways are modelled using an RTN the model is able to consider interconversion of energy into various forms. Therefore, it will be applied to assess the value transfer that could occur between different energy vectors.

\section{Acknowledgements}

We would like to acknowledge the Engineering and Physical Sciences Research Council for the financial support of the authors through the Whole Systems Energy Modelling Consortium (EP/K039326/1). We would like to thank Prof. Nilay Shah and everyone in the Consortium for their useful and stimulating discussions. We would also like to thank the two anonymous reviewers for their helpful and positive comments. 


\section{References}

[1] Energy Research Partnership. The future role for energy storage in the UK. Technical report, 2011.

[2] Institution of Mechanical Engineers. Energy storage: the missing link in the UK's energy commitments. Technical report, 2014.

[3] International Energy Agency. Energy roadmap: energy storage, http://www.iea.org/publications/freepublications/publication/technologyroadmapenergystorage.pdf, 2014. [Online; accessed 9-12-2014].

[4] P. Denholm, E. Ela, B. Kirby, and M. Milligan. The role of energy storage with renewable electricity generation. Technical report, National Renewable Energy Laboratory, 2010.

[5] Directorate-General for Energy. European Commission. The future role and challenges of energy storage, http://ec.europa.eu/energy/infrastructure/doc/energy-storage/2013/energy_storage.pdf, 2013. [Online; accessed 9-12-2014].

[6] J. Eyer and G. Corey. Energy storage for the electricity grid: benefits and market potential assessment guide. Technical report, Sandia National Laboratories, 2010.

[7] Electric Power Research Institute, Palo Alto, CA. Report 1020676: Electrical energy storage technology options, http://www.epri.com/abstracts/pages/productabstract.aspx?productid $=000000000001020676$, December 2010. [Online; accessed 9-12-2014].

[8] B. Dunn, H. Kamath, and J.-M. Tarascon. Electrical energy storage for the grid: a battery of choices. Science, 334:928-935, 2011.

[9] M. Ball and M. Wietschel. The future of hydrogen - opportunities and challenges. International Journal of Hydrogen Energy, 34:615-627, 2009.

[10] National Research Council. The hydrogen economy: opportunities, costs and barriers, and R\&D needs, http://books.nap.edu/openbook.php?isbn=0309091632, 2004. [Online; accessed 9-12-2014].

[11] S. Dunn. Hydrogen futures: toward a sustainable energy system. International Journal of Hydrogen Energy, 27:235-264, 2002.

[12] C. Thistlethwaite, R Jones, and N. Holmes. Potential for grid-scale storage of hydrogen in on and offshore concrete structures. Technical report, Scottish Hydrogen and Fuel Cell Association and University of Dundee, 2011.

[13] S. Jebaraj and S. Iniyan. A review of energy models. Renewable and Sustainable Energy Reviews, 10:281-311, 2006.

[14] Y. Zeng, Y. Cai, G. Huang, and J. Dai. A review on optimization modeling of energy systems planning and GHG emission mitigation under uncertainty. Energies, 4:1624-1656, 2011.

[15] S.C. Bhattacharyya. A review of energy system models. International Journal of Energy Sector Management, 4:494-518, 2010. 
[16] D. Connoly, H. Lund, B.V. Mathiesen, and M. Leahy. A review of computer tools for analysing the integration of renewable energy into various energy systems. Applied Energy, 87:1059-1082, 2010.

[17] R. Loulou, G. Goldstein, and K. Noble. Documentation for the MARKAL Family of Models, http://www.iea-etsap.org/web/mrkldoc-i_stdmarkal.pdf, 2004. [Online; accessed 9-12-2014].

[18] R. Loulou, U. Remne, A. Kanudia, A. Lehtila, and G. Goldstein. Documentation for the TIMES Model Part 1, http://www.iea-etsap.org/web/docs/timesdoc-intro.pdf, 2005. [Online; accessed 9-12-2014].

[19] S. Messner and M. Strubegger. User's Guide for MESSAGE III, http://www.iiasa.ac.at/Admin/PUB/Documents/WP-95-069.pdf., $1995 . \quad$ [Online; accessed 9-122014].

[20] D. Henning. MODEST: An energy-system optimisation model applicable to local utilities and countries. Energy, 22:1135-1150, 1997.

[21] D. Henning, S. Amiri, and K. Holmgren. Modelling and optimisation of electricity, steam and district heating production for a local Swedish utility. European Journal of Operational Research, 175:1224-1247, 2006.

[22] S. Samsatli, N. J. Samsatli, and N. Shah. BVCM: A comprehensive and flexible toolkit for whole system biomass value chain analysis and optimisation - Mathematical formulation. Applied Energy, 147:131-160, 2015.

[23] A. Almansoori and N. Shah. Design and operation of a future hydrogen supply chain. Chemical Engineering Research and Design, 84(A6):423-438, 2006.

[24] A. Almansoori and N. Shah. Design and operation of a future hydrogen supply chain: multiperiod model. International Journal of Hydrogen Energy, 34:7883-7897, 2009.

[25] A. Almansoori and N. Shah. Design and operation of a stochastic hydrogen supply chain network under uncertainty. International Journal of Hydrogen Energy, 37:3965-3977, 2012.

[26] A. Zamboni, N. Shah, and F. Bezzo. Spatially-explicit static model for the strategic design of future bioethanol production systems. 1. Cost minimisation. Energy Fuels, 23:5121-5133, 2009.

[27] A.J. Dunnett, C.S. Adjiman, and N. Shah. A spatially explicit whole-system model of the lignocellulosic bioethanol supply chain: an assessment of decentralised processing potential. Biotechnology for Biofuels, 1,2008 .

[28] M. Chaudry, N. Jenkins, Qadrdan M., and J.Z. Wu. Combined gas and electricity network expansion planning. Applied Energy, 113:1171-1187, 2014.

[29] D. Pudjianto, M. Aunedi, P. Djapic, and G. Strbac. Whole-systems assessment of the value of energy storage in low-carbon electricity systems. IEEE Transactions on Smart Grid, 5:1098-1109, 2014.

[30] N.J. Samsatli and M. Jennings. Optimization and systems integration. In J. Keirstead and N. Shah, editors, Urban Energy Systems: An Integrated Approach, chapter 9. Optimization and systems integration, pages 157-184. Routledge, 2011. 
[31] M.G. Hoffman, A. Sadovsky, M.C. Kintner-Meyer, and J.G. DeSteese. Analysis tools for sizing and placement of energy storage in grid applications. a literature review. Technical report, U.S. Department of Energy, 2010.

[32] A.I. Bejan, R.J. Gibbens, and F.P. Kelly. Statistical aspects of storage systems modelling in energy networks. In 46th Annual Conference on Information Sciences and Systems, 2012.

[33] A. Borghetti, C. D' Ambrosio, A. Lodi, and S. Martello. An MILP approach for short-term hydro scheduling and unit commitment with head-dependent reservoir. IEEE Transactions on Power Systems, 23:1115-1124, 2008.

[34] M.E. Khodayar, M. Shahidehpour, and L. Wu. Enhancing the dispatchability of variable wind generation by coordination with pumped-storage hydro units in stochastic power systems. IEEE Transactions on Power Systems, 28:2808-2818, 2013.

[35] M. Parastegari, R.-A. Hooshmand, A. Khodabakhshian, and A.-H. Zare. Joint operation of wind farm, photovoltaic, pump-storage and energy storage devices in energy and reserve markets. Electrical Power and Energy Systems, 64:275-284, 2015.

[36] G. Carpinelli, F. Mottola, D. Proto, and A. Russo. Optimal allocation of dispersed generators, capacitors and distributed energy storage systems in distribution networks. In Modern Electric Power Systems (MEPS), 2010 Proceedings of the International Symposium, 2010.

[37] M. Ghofrani, A. Arabali, M. Etezadi-Amoli, and M.S. Fadali. A framework for optimal placement of energy storage units within a power system with high wind penetration. IEEE Transactions on Sustainable Energy, 4(2):434-442, 2013.

[38] M. Nick, M. Hohmann, R. Cherkaoui, and M Paolone. Optimal location and sizing of distributed storage systems in active distribution networks. In PowerTech (POWERTECH), 2013 IEEE Grenoble, 2013.

[39] M. Geidl and G. Andersson. Optimal coupling of energy infrastructures. In IEEE Power Tech, 2007.

[40] M. Geidl, G. Koeppel, P Favre-Perrod, B. Klöckl, G. Andersson, and Frölich. Energy hubs for the future. IEEE Power Energy Mag, 5:24-30, 2007.

[41] A. Parisio, C. Del Vecchio, and A. Vaccaro. A robust optimization approach to energy hub management. International Journal of Electrical Power and Energy Systems, 42:98-104, 2012.

[42] C. Weber, J. Keirstead, N.J. Samsatli, N. Shah, and D. Fisk. Trade-offs between layout of cities and design of district energy systems. In 23rd International Conference on Efficiency, Cost, Optimization, Simulation, and Environmental Impact of Energy Systems, Lausanne, 2010.

[43] J. Keirstead and C. Calderon. Capturing spatial effects, technology interactions, and uncertainty in urban energy and carbon models: Retrofitting Newcastle as a case study. Energy Policy, 46:253-267, 2012.

[44] J. Keirstead, N.J. Samsatli, A.M. Panteleo, and N. Shah. Evaluating biomass energy strategies for a UK eco-town with an MILP optimization model. Biomass and Bioenergy, 39:306-316, 2012.

[45] NETA. The New Electricity Trading Arrangements. http://www.bmreports.com/. [Online; accessed 9-12-2014]. 
[46] Gridwatch. http://www.gridwatch.templar.co.uk/index.php. [Online; accessed 09-12-2014].

[47] E. Kondili, C.C. Pantelides, and R. W. H. Sargent. A general algorithm for short-term scheduling of batch-operations.1. MILP formulation. Computers \& Chemical Engineering, 17:211-227, 1993.

[48] C.C. Pantelides. Unified frameworks for the optimal process planning and scheduling. In Proceedings of the 2nd Conference on the Foundations of Computer Aided Operations, page 253. New York: Cache Publications, 1994.

[49] Richard Loulou. ETSAP-TIAM: the TIMES integrated assessment model. part II: mathematical formulation. Computational Management Science, 5(1-2):41-66, February 2008.

[50] Ramachandran Kannan. The development and application of a temporal \{MARKAL\} energy system model using flexible time slicing. Applied Energy, 88(6):2261-2272, 2011.

[51] W. A. Amos. Costs of storing and transporting hydrogen. Technical report, National Renewable Energy Laboratory, 1998.

[52] E.W. Weisstein. Moore Neighborhood. From MathWorld - A Wolfram Web Resource. http://mathworld.wolfram.com/MooreNeighborhood.html. [Online; accessed 9-12-2014].

[53] G. Saur and T. Ramsden. Wind electrolysis: hydrogen cost optimisation. Technical report, National Renewable Energy Laboratory, 2011.

[54] National Renewable Energy Laboratory. Current (2009) state-of-the-art hydrogen production cost estimate using water electrolysis. Technical report, 2009.

[55] S. Satyapal. Overview of hydrogen and fuel cell activities, http://www.iphe.net/docs/events/china_910/1-1_March 2010. [Online; accessed 9-12-2014].

[56] P. Withrington. Fact sheet 7, transport watch UK, www.transport-watch.co.uk. Online, September 2008. [Online; accessed 9-12-2014]. 


\section{Nomenclature}

Indices and sets

$\begin{array}{ll}b \in \mathbb{B} & \text { Transport infrastructures } \\ c \in \mathbb{C} & \text { Cells } \\ d \in \mathbb{D} & \text { Daily intervals } \\ f \in \mathbb{F} & \text { Flow directions } \\ h \in \mathbb{H} & \text { Hourly intervals } \\ \tilde{h} \in \tilde{\mathbb{H}} & \text { Contiguous hourly intervals } \\ i \in \mathbb{I} & \text { Performance metrics (e.g. costs, GHG emissions etc.) } \\ l \in \mathbb{L} & \text { Transport technologies } \\ p \in \mathbb{P} & \text { Production technologies } \\ r \in \mathbb{R} & \text { Resources } \\ s \in \mathbb{S} & \text { Storage technologies } \\ t \in \mathbb{T} & \text { Seasonal intervals } \\ y \in \mathbb{Y} & \text { Yearly intervals }\end{array}$

Parameters

$a_{s c}$

$b_{b}^{\max }$

Binary parameter: 1 if storage $s$ is allowed in cell $c ; 0$ otherwise

Maximum capacity of infrastructure $b[\mathrm{MWh} / \mathrm{h}]$

$B I_{b}$

$B R_{p}$

Unit capital impact for infrastructure $b[£ / \mathrm{km}]$

Maximum total number of technologies $p$ (build rate)

$d_{c c^{\prime}}$

$L B_{l b}$

$M I_{r i}$

$m_{r c}^{\max }$

$n_{d}^{d w}$

$n_{h}^{h d}$

$n_{t}^{w t}$

$n_{y}^{y y}$

$p_{p}^{\max }$

$p_{p}^{\min }$

$q_{l}^{\max }$

Distance between centres of cells $c$ and $c^{\prime}[\mathrm{km}]$

Binary parameter: 1 if transport technology $l$ can use infrastructure $b ; 0$ otherwise

Unit impact of importing resource $r$ [£/MWh]

Maximum rate of import of resource $r$ in cell $c[\mathrm{MWh} / \mathrm{h}]$

Number of times day type $d$ occurs in a week

Duration of hourly interval $h[\mathrm{~h}]$

Number of repeated weeks in season $t$

Number of repeated years in period $y$

Maximum production rate of technology $p[\mathrm{MWh} / \mathrm{h}]$

Minimum production rate of technology $p[\mathrm{MWh} / \mathrm{h}]$

Max transfer rate for each transport mode $l[\mathrm{MWh} / \mathrm{h}]$

$R P I_{\text {richdty }} \quad$ Unit purchase impact of resource $r$, e.g. from the grid, in cell $c$ during hour $h$, day $d$, season $t$ and year $y[£ / \mathrm{MWh}]$

$s_{s}^{\text {hold,max }} \quad$ Maximum storage capacity of a single storage facility $s$ [MWh]

$s_{s}^{\text {put,max }} \quad$ Maximum rate of addition to the storage $s[\mathrm{MWh} / \mathrm{h}]$

$s_{s}^{\text {get,max }} \quad$ Maximum rate of withdrawal from the storage $s[\mathrm{MWh} / \mathrm{h}]$

$S C I_{s i} \quad$ Unit capital impact of storage technology $s[£]$

$S P I_{s i}$

$S H I_{s i}$

$S G I_{s i}$

$T C I_{p i}$

$\hat{T} I_{l i}$

Unit operating impact for putting inventory into (charging) storage $s[£ / \mathrm{MWh}]$

Unit operating impact for holding (maintaining) inventory in storage $s[£ / \mathrm{MWh}]$

Unit operating impact for withdrawing inventory from storage $s[£ / \mathrm{MWh}]$

Unit capital and impact of technology $p[£]$

Unit impact for transportation $[£ / \mathrm{km} / \mathrm{MWh}]$ 


$\begin{array}{ll}\bar{T} I_{l i} & \text { Unit impact for transportation, indpendent of distance, e.g. flat rate of freight charges } \\ T O I_{p i} & {[£ / \mathrm{MWh}]} \\ u_{r c h d t y}^{\max } & \text { Unit operating impact of technology } p[£ / \mathrm{MWh}] \\ & \text { Maximum availability of resource } r \text { in cell } c \text { during hour } h \text {, day } d \text {, season } t \text { and year } y \\ X I_{r i} & {[\mathrm{MWh} / \mathrm{h}]} \\ x_{r c}^{\max } & \text { Unit impact of exporting resource } r[£ / \mathrm{MWh}] \\ x_{c} & \text { Maximum rate of export of resource } r \text { in cell } c[\mathrm{MWh} / \mathrm{h}] \\ y_{c} & \text { x-coordinate of the centroid of cell } c \\ \alpha_{r p} & \text { y-coordinate of the centroid of cell } c \\ \beta_{b} & \text { Conversion factor or resource } r \text { in technology } p \\ & \text { Indicates bidirectionality of transport network: }-1 \text { if unidirectional A to B only; } 0 \text { if } \\ \gamma_{i} & \text { independent bidirectional, i.e. A to B and B to A need separate links; } 1 \text { if bidirectional } \\ \nu_{c c^{\prime}} & \text { Capital charge factor } \\ \sigma_{s r f}^{\text {get }} & \text { Binary parameter, } 1 \text { if cell } c \text { is adjacent to cell } c^{\prime} \\ \sigma_{s r f}^{\text {hold }} & \text { Conversion factor when withdrawing resource } r \text { from storage } s \\ \sigma_{s r f}^{\text {put }} & \text { Conversion factor when holding resource } r \text { in storage } s \\ \bar{\tau}_{l r f} & \text { Conversion factor when putting resource } r \text { into storage } s \\ \hat{\tau}_{l r f} & \text { Distance-independent conversion factor for transport mode } l \text { transporting resource } r \\ \omega_{i} & \text { Distance-dependent conversion factor for transport mode } l \text { transporting resource } r \\ & \text { Weighting to each objective component }\end{array}$

Positive variables

$D_{\text {rchdty }}$

$I_{\text {schdty }}$

$I_{\text {scdty }}^{0, \text { act }}$

$I_{\text {scdty }}^{0, \text { sim }}$

$\mathscr{I}^{P}$

$\mathscr{I}_{i}^{Q}$

$\mathscr{I}_{i}^{S}$

$\mathscr{I}_{i}{ }^{m}$

$\mathscr{I}_{i}^{p}$

$\mathscr{I}_{i}^{q}$

$\mathscr{I}_{i}^{s}$

$\mathscr{I}_{i}^{u}$

$\mathscr{I}_{i}^{x}$

$M_{\text {rchdty }}$

$\mathscr{P}_{\text {pchdty }}$

$\mathscr{Q}_{l c c^{\prime} h d t y}$

Demand for resource $r$ in cell $c$ during hour $h$, day $d$, season $t$ and year $y[\mathrm{MWh} / \mathrm{h}]$

Inventory of storage $s$ in cell $c$ during hour $h$, day $d$, season $t$ and year $y$ [MWh]

Inventory of storage $s$ in cell $c$ at the start of day $d$, season $t$ and year $y$ [MWh]

Inventory of storage $s$ in cell $c$ at the start of the simulated cycle for day $d$, season t and year $y$ [MWh]

Total impact of building new conversion technologies

Total impact of building new transport infrastructures

Total impact of building new storage facilities

Total impact of importing resources abroad

Total impact of operating conversion technologies

Total impact of transporting resources

Total impact of operating storage facilities

Total impact of utilising locally-available resources

Total impact of exporting resources

Rate of import of resource $r$ in cell $c$ during hour $h$, day $d$, season $t$ and year $y[\mathrm{MWh} / \mathrm{h}$ ]

Total utilisation rate of technology $p$ in cell $c$ during hour $h$, day $d$, season $t$ and year $y$ $[\mathrm{MWh} / \mathrm{h}]$

Rate of transport via mode $l$ from cell $c$ to $c^{\prime}$ during hour $h$, day $d$, season $t$ and year $y$ $[\mathrm{MWh} / \mathrm{h}]$

$\mathscr{S}_{\text {sch }}^{\text {get }}$ ty

Rate at which resources is withdrawn from storage $s$ in cell $c$ during hour $h$, day $d$, season $t$ and year $y[\mathrm{MWh} / \mathrm{h}]$ 


$\mathscr{S}_{\text {schdty }}^{\text {hold }}$
$\mathscr{S}_{\text {schdty }}^{\text {put }}$
$U_{\text {rchdty }}$
$X_{r c h d t y}$
Free variabl
$P_{r c h d t y}$
$Q_{r c h d t y}$
$S_{r c h d t y}$
$Z$
$\delta_{s c d t y}$
$\delta_{s c t y}$
$\delta_{s c y}$

Binary variables

$B_{b c c^{\prime}}$

Integer variables

$N_{p c}^{P}$

$N_{s c}^{S}$

Abbreviations

$\mathrm{CGH}_{2}$

$\mathrm{CGH}_{2} \mathrm{~S}$

$\mathrm{LH}_{2}$

$\mathrm{LH}_{2} \mathrm{~S}$
Rate at which inventory is held in storage $s$ in cell $c$ during hour $h$, day $d$, season $t$ and year $y[\mathrm{MWh}]$

Rate at which resource is added to storage $s$ in cell $c$ during hour $h$, day $d$, season $t$ and year $y[\mathrm{MWh} / \mathrm{h}]$

Utilisation of resource $r$ in cell $c$ during hour $h$, day $d$, season $t$ and year $y[\mathrm{MWh} / \mathrm{h}]$

Rate of export of resource $r$ in cell $c$ during hour $h$, day $d$, season $t$ and year $y[\mathrm{MWh} / \mathrm{h}]$

Net rate of production of resource $r$ in cell $c$ during hour $h$, day $d$, season $t$ and year $y$ $[\mathrm{MWh} / \mathrm{h}]$

Net rate of transport of resource $r$ into cell $c$ from all other cells during hour $h$, day $d$, season $t$ and year $y[\mathrm{MWh} / \mathrm{h}]$

Net utilisation rate of stored resource $r$ in cell $c$ during hour $h$, day $d$, season $t$ and year $y[\mathrm{MWh} / \mathrm{h}]$

Objective function

Accumulation of inventory $s$ in cell $c$ during day $d$, season $t$ and year $y$ [MWh]

Accumulation of inventory $s$ in cell $c$ during season $t$ and year $y$ [MWh]

Accumulation of inventory $s$ in cell $c$ during year $y$ [MWh]

1 if transport infrastrusture $b$ is to be built between cells $c$ and $c^{\prime} ; 0$ otherwise

Number of technologies $p$ in cell $c$

Number of storage facilities $s$ in cell $c$

Compressed hydrogen gas

Compressed hydrogen gas storage

Liquid hydrogen

Liquid hydrogen storage 


\section{AppendixA. Derivation of the constraints linking different time periods}

Having applied the non-uniform hierarchical time discretisation, the model has a greatly reduced number of variables and constraints. In particular, the degrees of freedom for the operation of the network are reduced by defining a set of profiles that are repeated to give a highly periodic operation over the whole time horizon. However, the inventory profile for all stored resources needs to be tracked throughout the whole of the time horizon in order to ensure that the storage facilities are sized correctly. Therefore, from the reduced set of operational degrees of freedom in the model (production rates, transportation rates and storage rates) it is necessary to reconstruct the inventory profiles over the whole time horizon assuming periodic operation of the energy network. This is the purpose of constraints 22 to 25 , which are now derived in more detail.

Given the operation of the network over the hourly intervals $h$, for a particular day type $d$ in season $t$ during yearly interval $y$, the inventory profile can be generated using constraints 15 to 17 provided the inventory at the start of the day, $I_{\text {scdty }}^{0, \text { sim }}$, is known. The change in inventory over the day type $d$ is the final inventory $I_{s c|\mathbb{H}| d t y}$ minus the initial inventory $I_{s c d t y}^{0, \text { sim }}$, which is equal to the variable $\delta_{s c d t y}$ (equation 18). If $I_{s c d t y}^{0, \text { sim }}$ were equal to the initial inventory at the start of the first occurrence of day type $d$ in the first week of season $t$ for the first year in yearly interval $y$, which we defined as $I_{\text {scdty }}^{0, \text { act }}$, then the inventory for any occurrence of day of type $d$ in the first week in season $t$ and first year of yearly interval $y$ can be obtained by adding $\delta_{\text {scdty }}$ for each additional occurrence. In particular, the starting inventory of the first occurrence of the second day type is given by $I_{s c 2 t y}^{0, \text { act }}=I_{s c 2 t y}^{0, \text { act }}+n_{1}^{d w} \delta_{s c 1 t y}$, which is a special case of equation 22 , for $d=1$. Similarly, $\delta_{s c t y}$ shifts the inventory forward one week at a time for season $t$ and yearly interval $y ; \delta_{s c y}$ shifts the inventory forward by one year (although the optional constraint 21 ensures that the inventory returns to its starting point after one year).

If the inventory variables in the model, $I_{\text {schdty }}$, represented the inventory in the first occurrence of each day type, $d$, in the first week of season $t$ and so on, then it is simple to obtain the inventory for any hour in the year using combinations of $\delta_{s c d t y}, \delta_{s c t y}$ and $\delta_{s c y}$. Thus one could write constraints for each hour to ensure that the storage capacity is never exceeded and that it does not fall below zero (e.g. constraints 26 to 33 and their counterparts). Note that while $I_{\text {schdty }}$ are positive variables, the actual inventory could still be negative if some of the $\delta$ variables are negative. However, the inventory profile over the first occurrence of any day type, week and year is not a good representation of the energy system in general. This is because the inventory levels may have associated with them a resource requirement and an operating cost: e.g. holding liquified natural gas or hydrogen in storage requires cooling in order to re-condense any boil-off and this can be modelled explicitly by setting $\sigma_{s r \text { dst }}^{\text {hold }}$ for the resource required to maintain the storage level and/or by providing an appropriate operating cost $\left(S H I_{s i}\right)$. Both the resource requirement and the operating cost are assumed to be proportional to the inventory level (see equations 14 and 47 and note that $\mathscr{S}_{\text {schdty }}^{\text {hold }}$ is proportional to $I_{\text {schdty }}$ ). The $\delta$ variables are positive, then the inventory levels increase each day and each week, so that the inventory levels will be lower in the first occurrence of each day type and week than the average inventory levels and therefore underestimate the resource requirements and/or costs; conversely, if the $\delta$ variables are negative, the inventory levels will overestimate the resource requirements and/or costs. Therefore, a better approximation is to consider an occurrence of each day type, week and year for each combination of $d, t$ and $y$ that results in the average inventories over all occurrences, weeks and years. This

is the origin of the $I_{\text {scdty }}^{0, \text { sim }}$ variables: $I_{\text {scdty }}^{0, \text { act }}$ is the initial inventory for the first occurrence of each day in day type $d$, first week in $t$ and first year in $y ; I_{s c d t y}^{0, \text { sim }}$ is the initial inventory for the "simulated" occurrence of each 
day, week and year. The inventory variables, $I_{\text {schdty }}$, relate to this simulated scenario, which for odd $n_{d}^{d w}$, $n_{t}^{w t}$ and $n_{y}^{y y}$ is the central occurrence of each day, week and year.

Consider just the set of occurrences for day type $d$, for the first week in season $t$ and the first year in yearly interval $y$. The average initial inventory over all $n_{d}^{d w}$ occurrences of day type $d,\left\langle I_{s c d t y}^{0, \text { act }}\right\rangle$, is given by:

$$
\begin{aligned}
\left\langle I_{s c d t y}^{0, \text { act }}\right\rangle & =\frac{I_{s c d t y}^{0, \text { act }}+\left[I_{s c d t y}^{0, \text { act }}+\delta_{s c d t y}\right]+\cdots+\left[I_{s c d t y}^{0, \text { act }}+\left(n_{d}^{d w}-1\right) \delta_{s c d t y}\right]}{n_{d}^{d w}} \\
& =I_{\text {scdty }}^{0, \text { act }}+\frac{\delta_{s c d t y}}{n_{d}^{d w}} \sum_{j=1}^{n_{d}^{d w}-1} j \\
& =I_{s c d t y}^{0, \text { act }}+\frac{\delta_{s c d t y}}{n_{d}^{d w}} \frac{\left(n_{d}^{d w}-1\right) n_{d}^{d w}}{2} \\
& =I_{\text {scdty }}^{0, \text { act }}+\delta_{s c d t y} \frac{n_{d}^{d w}-1}{2}
\end{aligned}
$$

Similarly, the average initial inventory over all weeks in season $t$ and all years in interval $y$ is:

$$
\left\langle I_{s c d t y}^{0, \text { act }}\right\rangle=I_{s c d t y}^{0, \text { act }}+\delta_{s c d t y} \frac{n_{d}^{d w}-1}{2}+\delta_{s c t y} \frac{n_{t}^{w t}-1}{2}+\delta_{s c y} \frac{n_{y}^{y y}-1}{2}
$$

Setting $I_{\text {scdty }}^{0, \text { sim }}$ equal to $\left\langle I_{\text {scdty }}^{0, \text { act }}\right\rangle$ results in equation 25.

Finally, while the $I_{\text {schdty }}$ variables refer to an average scenario, the inventory constraints must relate to the actual inventories. As discussed earlier, due to the assumption of periodicity, the inventory levels will either progressively increase or decrease within a particular day $d$, season $t$ and year $y$. Therefore, it is sufficient to constrain only the inventories for the first and last occurrence of each day type, the first and last week of each season and the first and last year of each yearly interval. Adding or subtracting $\delta_{s c d t y}\left(n_{d}^{d w}-1\right) / 2$ gives the inventory in the last or first occurrence of day type $d$, respectively. Adding or subtracting $\delta_{s c t y}\left(n_{t}^{w t}-1\right) / 2$ gives the last or first week in season $t$. Adding or subtracting $\delta_{s c y}\left(n_{y}^{y y}-1\right) / 2$ shifts to the last or first year. Taking all eight combinations of these results in constaints 26 to 33 . 